Y. Yoshino

Nagoya Math. J.

Vol. 103 (1986), 95-125

\title{
TOWARD THE CONSTRUCTION OF BIG COHEN-MACAULAY MODULES
}

\author{
YUJI YOSHINO
}

\section{§. Introduction}

What we call the homological conjectures on commutative Noetherian local rings were first collected and partially settled by C. Peskine and L. Szpiro $\left[\mathrm{PS}_{1}\right]$. The subsequent remarkable progress was made by $\mathrm{M}$. Hochster $\left[\mathrm{H}_{1}\right]$ who conjectured the existence of big Cohen-Macaulay modules and solved it in the affirmative for equicharacteristic local rings. It is, however, still open in general setting.

If every local ring has a big Cohen-Macaulay module, then all the homological conjectures will be automatically proved as argued in $\left[\mathrm{H}_{1}\right]$. This makes us feel that it should be big Cohen-Macaulay modules that we must preferentially consider. It would be also desirable to build a characteristic free way to the homological conjectures. In fact, though there exist various other papers concerning the homological conjectures such as $\left[\mathrm{H}_{2}\right],\left[\mathrm{H}_{3}\right],\left[\mathrm{H}_{4}\right],\left[\mathrm{H}_{5}\right],[\mathrm{F}],[\mathrm{I}],\left[\mathrm{PS}_{2}\right],\left[\mathrm{R}_{1}\right],\left[\mathrm{R}_{2}\right],\left[\mathrm{Y}_{1}\right]$ etc., one finds that most of their argument heavily depend on the characteristic of local rings.

The main purpose of the present paper is to provide several new tools for the conjecture which seem to meet our demands. Principal results are stated in Theorem (3.6) and Theorem (3.11). Roughly speaking Theorem (3.6) reduces the existence problem of big Cohen-Macaulay modules to a problem for Artinian local rings, and Theorem (3.11) shows that if big CohenMacaulay modules always exist, then there are "universal" ones in some sense.

The idea of the proof of Theorem (3.6) is the following: Let $(R, \mathrm{~m})$ be a local ring and let $\left\{q_{i} \mid i \in \mathbb{N}\right\}$ be a descending sequence of m-primary ideals such that $R=\varliminf R / \mathfrak{q}_{i}$. Assume that $M_{i}$ is given as an $R / \mathfrak{q}_{i}$-module for each $i \in \mathbb{N}$. If each $M_{i}$ is "good enough", and if they form an inverse system of $R$-modules, then $\underline{\lim } M_{i}$ will be a "good enough" $R$-module again,

Received March 1, 1985. 
which might be possibly Cohen-Macaulay. However it often happens that $M_{i}$ has no relation with $M_{j}$ if $i \neq j$. In this case how can one construct an $R$-module which may be reasonably considered as a limit of the $M_{i}$ ? If such construction of limit is always possible, then the existence of "good" modules over $R / \mathfrak{q}_{i}(i \in \mathbb{N})$ will automatically imply the existence of corresponding "good" ones over $R$ including big Cohen-Macaulay modules.

In Section 1 we develope the method of constructing limit of modules which enables us to realize the above idea. We refer to such a limit as a separated ultraproduct because of its resemblance to ultraproduct. Main fact concerning it is the Exactness Theorem (1.18). It will make it possible to compute various examples of separated ultraproducts.

Section 2 is mainly concerned with the problem for Artinian local rings which corresponds, by the reduction in the above idea, to the existence problem of big Cohen-Macaulay modules for local rings of high dimension. An important role will be played by those algebras which the author names rich algebras and poor algebras. The reader should pay attention to the existence of generic poor algebras (2.10), where he will notice that the author was much inspired by the method of Hochster's in $\left[\mathrm{H}_{1}\right]$.

Section 1 and Section 2 can be read independently.

In Section 3 we prove the main theorems (3.6) and (3.11), whose proofs are the core of this paper.

Although he could not settle the existence problem of big CohenMacaulay modules, the author believes that the raison d'etre of this paper lies in providing several new concepts and methods in the theory of commutative algebras.

\section{$\S 1$. Separated ultraproducts}

We first recall some fundamental concepts about ultrafilters.

Let $N$ be an infinite set of indices, which is in many cases the set of natural numbers $\mathbb{N}$. A filter on $N$ is a nonempty family $\widetilde{F}$ of subsets of $N$ satisfying (i) $\phi \notin \mathfrak{F}$, (ii) if $A, B \in \mathfrak{F}$ then $A \cap B \in \widetilde{F}$ and (iii) if $A \in \mathfrak{F}$ and $A \subset B \subset N$ then $B \in \mathfrak{F}$. A filter $\widetilde{\mho}$ on $N$ is principal if there is an $a \in N$ such that $\mathfrak{F}=\{B \mid a \in B \subset N\}$, otherwise it is called nonprincipal. The Fréchet filter on $N$ is a filter consisting of cofinite subsets of $N$, i.e. $\{A \mid N-A$ is finite $\}$.

An ultrafilter $\mathfrak{F}$ on $N$ is a filter on $N$ which is maximal with respect to inclusion in the class of all filters on $N$. It is clear from Zorn's lemma that for any filter $\mathscr{F}$ there exists an ultrafilter on $N$ which contains $\mathfrak{F}$. It is 
also easy to see the following lemmas.

(1.1) For a filter $\mathfrak{F}$ on $N$, the following conditions are equivalent.

(i) $\widetilde{\mho}$ is an ultrafilter.

(ii) $A \notin \mathfrak{F}$ implies $N-A \in \mathfrak{F}$.

(iii) If $\bigcup_{i=1}^{n} A_{i} \in \mathfrak{F}$ for $A_{i} \subset N(i=1,2, \cdots, n)$, then one of the $A_{i}$ is an element of $\mathfrak{S}$.

(1.2) For an ultrafilter $\mathfrak{F}$ on $N$, the following conditions are equivalent.

(i) $\widetilde{F}$ is nonprincipal.

(ii) $\widetilde{\mho}$ contains the Fréchet filter on $N$.

(iii) No finite subset of $N$ belongs to $\mathfrak{F}$.

In particular there always exists a nonprincipal ultrafilter on $N$.

An ultrafilter $\widetilde{\mho}$ on $N$ is said to be $\omega$-incomplete if there are $A_{i} \in \widetilde{\mho}$ $(i \in \mathbb{N})$ such that $\bigcap_{i \in \mathbb{N}} A_{i} \notin \widetilde{F}$.

(1.3) For an ultrafilter $\widetilde{\&}$ the following are equivalent.

(i) $\widetilde{\mho}$ is $\omega$-incomplete.

(ii) There is a countable set $\left\{B_{i} \in \mathfrak{F} \mid i \in \mathbb{N}\right\}$ such that $B_{i+1} \subset B_{i}(i \in \mathbb{N})$ and $\bigcap_{i \in \mathrm{N}} B_{i}=\phi$.

(iii) There is a mapping $f: N \rightarrow \mathbb{N}$ such that $\{i \in N \mid f(i) \geqq j\} \in \widetilde{F}$ for any $j \in \mathbb{N}$.

By this lemma we easily see the following.

(1.4) An $\omega$-incomplete ultrafilter $\mathfrak{F}$ on $N$ is always nonprincipal. If $N$ is a countable set, then the converse is also true.

A usual argument using Zorn's lemma leads us to the following.

(1.5) If $A$ is an infinite subset of $N$, then there is an $\omega$-incomplete ultrafilter $\mathfrak{F}$ such that $A \in \widetilde{F}$. In particular there always exists an $\omega$-incomplete ultrafilter on $N$.

In the rest of this paper we always assume that $\mathfrak{\Im}$ is an $\omega$-incomplete (hence nonprincipal) ultrafilter on $N$.

For the simplicity of notation, we make the following

(1.6) Definition. Let $\{P(i) \mid i \in N\}$ be a family of propositions indexed by $N$. We say that $P(i)$ holds for almost all $i \in N$ with respect to $\mathfrak{F}$ (abbreviation; $P(i)$ for a.a. $i \in N)$ if $\{i \in N \mid P(i)$ is true $\} \in \mathfrak{F}$. Note that if $P(i)$ holds for a.a. $i \in N$ then $P(i)$ actually holds for infinitely many $i \in N$. (See (1.1) and (1.2).) However the converse is not true in general.

Let $\left\{R_{i} \mid i \in N\right\}$ be a set of local rings indexed by $N$ and let $\mathfrak{m}_{i}$ be the maximal ideal of $R_{i}$ for each $i \in N$. In this section we shall be concerned 
exclusively with the separated ultraproducts of modules over $R_{i}$ which is defined by the following

(1.7) Definition. Let $M_{i}$ be an $R_{i}$-module for each $i \in N$. Then we set

$$
\prod_{i \in N} M_{i}:=\prod_{i \in N} M_{i} / \sim
$$

where $\sim$ is the relation defined as follows: $\left(a_{i}\right)_{i \in N} \sim\left(b_{i}\right)_{i \in N}$ if and only if, for any integer $n, a_{i}-b_{i} \in \mathfrak{m}_{i}^{n} M_{i}$ for a.a: $i \in N$. $\tilde{\prod}_{i \in N} M_{i}$ is called the separated ultraproduct of the $M_{i}$. We denote by $\left(a_{i}\right)_{i \in N}$ the class of $\left(a_{i}\right)_{i \in N^{*}}$ in $\tilde{\prod}_{i \in N} M_{i}$.

Note that if all $R_{i}$ are fields, then this notion coincides with the usual ultraproduct of vector spaces.

(1.8) Remark. (i) It is easy to see that $\tilde{R}:=\tilde{\prod}_{i \in N} R_{i}$ forms a ring by putting $\left(a_{i}\right)_{i \in N}+\left(b_{i}\right)_{i \in N}=\left(a_{i}+b_{i}\right)_{i \in N}$ and $\left(a_{i}\right)_{\tilde{i} \in N}\left(b_{i}\right)_{\tilde{i} \in N}=\left(a_{i} b_{i}\right)_{\tilde{i} \tilde{H}_{N}}$. Note that $(0)_{i \in N}$ (resp. $(1)_{i \in N}$ ) is a null element (resp. a unity) of $\tilde{R}$. Also note that $\tilde{R}$ is a quasi-local ring with the maximal ideal $\tilde{\mathfrak{m}}:=\left\{\left(a_{i}\right)_{i \in N} \mid a_{i} \in \mathfrak{m}_{i}\right.$ for a.a. $i \in N\}$. In fact if $\left(a_{i}\right)_{i \in N} \notin \tilde{\mathfrak{m}}$ then $a_{i} \notin \mathfrak{m}_{i}$ for a.a. $i \in N$ by (1.1) and hence there exist $b_{i} \in R_{i}(i \in N)$ such that $a_{i} b_{i}=1$ for such $i$. This shows that $\left(a_{i}\right)_{i \in N}\left(b_{i}\right)_{i \in N}=(1)_{i \in N}$ in $\tilde{R}$. This proves that $\tilde{\mathfrak{m}}$ is the unique maximal ideal of $\tilde{R}$. Remark that $\tilde{R} / \widetilde{\mathfrak{m}} \cong \prod_{i \in N}^{*}\left(R_{i} / \mathfrak{m}_{i}\right)$ where $\prod_{i \in N}^{*}$ stands for the usual ultraproduct of fields.

(ii) $\tilde{M}:=\tilde{\prod}_{i \in N} M_{i}$ is an $\tilde{R}$-module by defining the $\tilde{R}$-action as follows; $\left(a_{i}\right)_{i \in N}\left(x_{i}\right)_{i \in N}=\left(a_{i} x_{i}\right)_{i \in N}$ for $a_{i} \in R_{i}$ and $x_{i} \in M_{i}(i \in N)$. If $f_{i}$ is an $R_{i}$-module homomorphism of $M_{i}$ to $M_{i}^{\prime}$ for all $i \in N$, then we naturally obtain the $\tilde{R}$-module homomorphism $\tilde{f}: \tilde{\prod}_{i \in N} M_{i} \rightarrow \widetilde{\prod}_{i \in N} M_{i}^{\prime}$ by setting $f\left(\left(x_{i}\right)_{i \in N}\right)=$ $\left(f_{i}\left(x_{i}\right)\right)_{i \in N}$.

(iii) If $x_{i}$ is an element of $M_{i}$ for a.a. $i \in N$ (not necessarily for all $i \in N$ ), then we can give the element $\left(x_{i}\right)_{i \in N}$ of $\tilde{\prod}_{i \in N} M_{i}$ without any ambiguity.

(iv) $\tilde{\Pi}_{i \in N} M_{i}$ is trivial if and only if $\mathfrak{m}_{i} M_{i}=M_{i}$ for a.a. $i \in N$.

(v) Let $\left(R_{i}^{\prime}, \mathfrak{m}_{i}^{\prime}\right) \rightarrow\left(R_{i}, \mathfrak{m}_{i}\right)$ be a local homomorphism for each $i \in N$. Assume that there is an integer $n$ such that $\mathfrak{m}_{i}^{n} \subset \mathfrak{m}_{i}^{\prime} R_{i}$ for a.a. $i \in N$. Then by definition the separated ultraproduct of the $M_{i}$ as $R_{i}$-modules coincides with the one as $R_{i}^{\prime}$-modules.

(1.9) Definition. A family of local rings $\left\{R_{i} \mid i \in N\right\}$ is said to be good if there exists an integer $n$ such that the maximal ideal $\mathfrak{m}_{i}$ is generated by at most $n$ elements in $R_{i}$ for a.a. $i \in N$. 
In most cases we restrict ourselves to consider a good family of local rings. A reasonable ground to do so lies in the following

(1.10) Lemma. Let $\left\{R_{i} \mid i \in N\right\}$ be a good family of local rings and let $M_{i}$ be an $R_{i}$-module for each $i \in N$. For an integer $j$, if $x_{i} \in \mathfrak{m}_{i}^{j} M_{i}$ for a.a. $i \in N$, then it holds that $\left(x_{i}\right)_{i \in N} \in \tilde{\mathfrak{m}}^{j} \tilde{M}$.

In fact since $\left\{R_{i}\right\}$ is good, there is an integer $n$ such that $\mathfrak{m}_{i}^{j}$ is generated by $n$ elements, say $\mathfrak{m}_{i}^{j}=\left(a_{i}(1), a_{i}(2) \cdots, a_{i}(n)\right) R_{i}$, for a.a. $i \in N$. Then $x_{i}$ $=\sum_{k=1}^{n} a_{i}(k) y_{i}(k)\left(y_{i}(k) \in M_{i}, k=1,2, \cdots, n\right)$ for a.a. $i \in N$. Thus by definition one sees that $\left(x_{i}\right)_{i \in N}=\sum_{k=1}^{n}\left(a_{i}(k)\right)_{i \in N}\left(y_{i}(k)\right)_{i \in N} \in \widetilde{\mathfrak{m}}^{j} \tilde{M}$.

Note that this is not true unless $\left\{R_{i}\right\}$ is good. For instance let $N=$ $\mathbb{N}$ and $R_{i}=M_{i}=K\left[\left[x_{1}, x_{2}, \cdots, x_{i}\right]\right]$ a formal power series ring over a field $K$ for all $i \in \mathbb{N}$ where $\operatorname{char}(K) \neq 2$. If we take elements $y_{i}:=x_{1}^{2}+x_{2}^{2}+$ $\cdots+x_{i}^{2} \in R_{i}(i \in \mathbb{N})$, then obviously $y_{i} \in \mathfrak{m}_{i}^{2}$ for all $i \in \mathbb{N}$, however we can see that $\left(y_{i}\right)_{i \in \mathbb{N}} \notin \tilde{\mathfrak{m}}^{2}$. In fact if $\left(y_{i}\right)_{i \in \mathbb{N}} \in \widetilde{\mathfrak{m}}^{2}$ then there would be finite elements $\left(\boldsymbol{z}_{i}(k)\right)_{i \in \mathbb{N}},\left(w_{i}(k)\right)_{i \in \mathbb{N}}$ in $\tilde{\mathfrak{m}}(k=1,2, \cdots, r)$ such that $\left(y_{i}\right)_{i \in \mathbb{N}}=$ $\sum_{k=1}^{r}\left(z_{i}(k)\right)_{i \in \mathbb{N}}\left(w_{i}(k)\right)_{i \in \mathbb{N}}$, hence it holds that $y_{i}=\sum_{k=1}^{r} z_{i}(k) w_{i}(k) \bmod \mathfrak{m}_{i}^{3}$ for a.a. $i \in \mathbb{N}$. In particular this equality would hold for some $i>2 r$, then

$$
2 x_{k}=\partial y_{i} / \partial x_{k} \in\left(z_{i}(1), z_{i}(2), \cdots, z_{i}(r), w_{i}(1), w_{i}(2), \cdots, w_{i}(r)\right)
$$

in $R_{i}$ for $k=1,2, \cdots, i$, therefore $\left(x_{1}, x_{2}, \cdots, x_{i}\right) R_{i}=\left(z_{i}(1), z_{i}(2), \cdots, z_{i}(r)\right.$, $\left.w_{i}(1), w_{i}(2), \cdots, w_{i}(r)\right) R_{i}$ for $\operatorname{char}(K) \neq 2$. This contradicts $i>2 r$.

The following proposition is essentially due to [BDLD; $\S 1$ (iii)].

(1.11) Proposition. If $\left\{R_{i} \mid i \in N\right\}$ is a good family of local rings and if $M_{i}$ is an $R_{i}$-module for $i \in N$, then $\tilde{M}:=\tilde{\Pi}_{i \in N} M_{i}$ is complete and separated with respect to the $\overline{\mathfrak{m}}$-adic topology.

Proof. We first prove the separatedness of $\tilde{M}$. Take any $\left(x_{i}\right)_{i \in N}$ in $\bigcap_{n=1}^{\infty} \tilde{\mathfrak{m}}^{n} \tilde{M}$. For any integer $n,\left(x_{i}\right)_{i \in N}$ may be written in the form

$$
\sum_{j=1}^{r}\left(a_{i}(j)\right)_{i \in N}^{\sim}\left(y_{i}(j)\right)_{i \in N}^{\sim}
$$

where

$$
\left(a_{i}(j)\right)_{i \in N} \in \overline{\mathfrak{m}}^{n},\left(y_{i}(j)\right)_{i \in N} \in \tilde{M}, j=1,2, \cdots, r .
$$

Then by definition one sees that

$$
x_{i}-\sum_{j=1}^{r} a_{i}(j) y_{i}(j) \in \mathfrak{m}_{i}^{n} M_{i}
$$


for a.a. $i \in N$, hence $x_{i} \in \mathfrak{m}_{i}^{n} M_{i}$ for a.a. $i \in N$. This shows that $\left(x_{i}\right)_{i \in N}=0$ in $\tilde{M}$.

Next we prove the completeness of $\tilde{M}$. Let $\left\{x(j)=\left(x_{i}(j)\right)_{i \in N} \mid j \in \mathbb{N}\right\}$ be a sequence in $\tilde{M}$ satisfying $x(j+1)-x(j) \in \tilde{\mathfrak{m}}^{j} \tilde{M}$ for any $j \in \mathbb{N}$. We would like to show the existence of $z \in \tilde{M}$ which satisfies $\left(^{*}\right) z-x(j) \in \tilde{\mathfrak{m}}^{j} \tilde{M}$ for any $j \in \mathbb{N}$. By (1.3) we have a mapping $f: N \rightarrow \mathbb{N}$ such that, for any $j \in$ $\mathbb{N}, f(i) \geqq j$ for a.a. $i \in N$. On the other hand it follows by definition that there are $A_{k} \in \widetilde{F}(k \in \mathbb{N})$ such that $x_{i}(k)-x_{i}(j) \in \mathfrak{m}_{i}^{j} M_{i}$ for $i \in A_{k}$ and $1 \leqq j \leqq k$, where we may choose $A_{0}=N$. Define a mapping $g: N \rightarrow$ $\mathbb{N} \cup\{\infty\}$ by $g(i):=\sup \left\{k \in \mathbb{N} \mid \cap_{j=0}^{k} A_{j} \ni i\right\}$. Note that $i \in A_{j}$ for all $j \leqq g(i)$ and that, for any $j \in \mathbb{N}, g(i) \geqq j$ for a.a. $i \in N$. Hence if we denote $h(i):=$ $\inf \{f(i), g(i)\}$ for $i \in N$, then $h$ is a mapping of $N$ to $\mathbb{N}$ and it satisfies that $i \in A_{h(i)}$ and that, for any $j \in \mathbb{N}, h(i) \geqq j$ for a.a. $i \in N$. Now set $z_{i}=x_{i}(h(i))$ and $z=\left(z_{i}\right)_{i \in N} \in \tilde{M}$. We prove that this $z$ satisfies $\left(^{*}\right)$. Let $j$ be an arbitrary positive integer. Then by the above there exists $B_{i} \in \mathfrak{F}$ such that $i \in A_{h(i)}$ and $h(i) \geqq j$ if $i \in B_{j}$. This implies that $z_{i}-x_{i}(j) \in \mathfrak{m}_{i}^{j} M_{i}$ for $i \in B_{j}$, whence by (1.10) we see that $z-x(j) \in \tilde{\mathfrak{m}}^{j} \tilde{M}$.

(1.12) Proposition. If $\left\{R_{i} \mid i \in N\right\}$ is a good family of local rings, then $\tilde{R}=\tilde{\Pi}_{i \in N} R_{i}$ is a complete Noetherian local ring. Moreover if there is an integer $r$ such that $\operatorname{emb}\left(R_{i}\right)=r$ for a.a. $i \in N$, then $\operatorname{emb}(\tilde{R})=r$.

Proof. Since $\tilde{R}$ is a complete and separated quasi-local ring by (1.11), in order to prove that $\tilde{R}$ is Noetherian it is sufficient to show the finiteness of $\operatorname{emb}(\tilde{R})$. (See [Ma; (28. P) Corollary 1].) Since $\left\{R_{i}\right\}$ is a good family, there is an integer $r$ such that $\mathfrak{m}_{i}$ is generated by $r$ elements for a.a. $i \in N$. Denote $\mathfrak{m}_{i}=\left(x_{i}(1), x_{i}(2), \cdots, x_{i}(r)\right) R_{i}$ for those $i \in N$ and $x(k)=\left(x_{i}(k)\right)_{i \in N}$ $(k=1,2, \cdots, r)$. It is easy to see that $\tilde{\mathfrak{m}}$ is actually generated by $x(1)$, $x(2), \cdots, x(r)$.

Conversely assume that $\tilde{\mathfrak{m}}$ is generated by $r$ elements, i.e. $\tilde{\mathfrak{m}}=(x(1)$, $x(2), \cdots, x(r)) \tilde{R}$ where $x(k)=\left(x_{i}(k)\right)_{i \in N}, k=1,2, \cdots, r$. Let $I_{i}$ be an ideal of $R_{i}$ generated by $x_{i}(1), x_{i}(2), \cdots, x_{i}(r)$. We prove that $\mathrm{m}_{i}=I_{i}$ for a.a. $i \in N$, hence that $m_{i}$ is generated by $r$ elements for a.a. $i \in N$. Suppose that this is not true. Then by (1.1) $I_{i}+\mathfrak{m}_{i}^{2} \neq \mathfrak{m}_{i}$ for a.a. $i \in N$ and thus we can find elements $y_{i} \in \mathfrak{m}_{i}-\left(I_{i}+\mathfrak{m}_{i}^{2}\right)$ for those $i \in N$. It obviously follows that $\left(y_{i}\right)_{i \in N}$ $\notin(x(1), x(2), \cdots, x(r)) \tilde{R}=\tilde{\mathfrak{m}}$, and hence $y_{i} \notin \mathfrak{m}_{i}$ for a.a. $i \in N$. This contradiction proves the proposition.

Note that the example after (1.10) shows that Propositions (1.11) and 
(1.12) fail unless $\left\{R_{i}\right\}$ is good.

Next we consider the dimension of $\tilde{R}$. For this, denote $h(R):=$ $\inf \left\{n \in \mathbb{N} \mid \mathfrak{m}^{n}\right.$ is contained in a parameter ideal of $\left.R\right\}$ for a local ring $(R, \mathfrak{m})$. The following result is proved by C. Mattescu and D. Popescu.

(1.13) Proposition. ([MP; (2.7)]). Let $\left\{R_{i}\right\}$ be a good family of local rings. Assume that

(i) there is an integer $d$ such that $\operatorname{dim}\left(R_{i}\right)=d$ for a.a. $i \in N$,

(ii) there is an integer $h$ such that $h\left(R_{i}\right) \leqq h$ for a.a. $i \in N$.

Then $\operatorname{dim}(\tilde{R})=d$.

Proof. Let $A=\left\{i \in N \mid \operatorname{dim}\left(R_{i}\right)=d, h\left(R_{i}\right) \leqq h\right\} \in \widetilde{F}$. For any $i \in A$ we may choose a system of parameters $x_{i}(1), x_{i}(2), \cdots, x_{i}(d)$ for $R_{i}$ satisfying $\mathfrak{m}_{i}^{h} \subset\left(x_{i}(1), x_{i}(2), \cdots, x(d)\right) R_{i}$. Denote $x(k)=\left(x_{i}(k)\right)_{i \in N}(k=1,2, \cdots, d)$. Then by definition it follows that $\tilde{\mathfrak{m}}^{h} \subset(x(1), x(2), \cdots, x(d)) \tilde{R}$, and this proves $\operatorname{dim}(\tilde{R}) \leqq d$. Next assume that $\tilde{\mathfrak{m}}^{n} \subset(z(1), z(2), \cdots, z(t)) \tilde{R}$ for some $n$ and $t \in \mathbb{N}$ and $z(k)=\left(z_{i}(k)\right)_{i \in N}(k=1,2, \cdots, t)$. It is sufficient to show that $t \geqq d$. By definition it holds that $\mathfrak{m}_{i}^{n} \subset\left(z_{i}(1), z_{i}(2), \cdots, z_{i}(t)\right) R_{i}+\mathfrak{m}_{i}^{n+1}$ for a.a. $i \in N$, hence for these $i \in N, \mathfrak{m}_{i}^{n} \subset\left(z_{i}(1), z_{i}(2), \cdots, z_{i}(t)\right) R_{i}$. Since $\operatorname{dim}\left(R_{i}\right)$ $=d$ for a.a. $i \in N$, we obtain that $t \geqq d$. This completes the proof.

(1.14) CoRollary. If $\left\{R_{i}\right\}$ is a good family of regular local rings satisfying $\operatorname{dim}\left(R_{i}\right)=d$ for a.a. $i \in N$, then $\tilde{R}=\tilde{\prod}_{i \in N} R_{i}$ is also a regular local ring of dimension $d$.

Proof. Since $R_{i}$ is a regular local ring for each $i \in N$, one has the equality $\operatorname{dim}\left(R_{i}\right)=\operatorname{emb}\left(R_{i}\right)=d$ for a.a. $i \in N$. Then it is obtained from (1.12) and (1.13) that $\operatorname{dim}(\tilde{R})=\operatorname{emb}(\tilde{R})=d$.

In many cases the dimension of $\tilde{\prod}_{i \in N} R_{i}$ is much different from each $\operatorname{dim}\left(R_{i}\right)$.

(1.15) Lemma. Let $(R, \mathfrak{m})$ be a local ring and $\left\{I_{i} \mid i \in N\right\}$ be a family of ideals of $R$ indexed by $N$ and denote $R_{i}=R / I_{i}(i \in N)$. Assume that for any $n \in \mathbb{N}, I_{i} \subset \mathfrak{m}_{i}^{n}$ for a.a. $i \ni N$. Then $\tilde{\Pi}_{i \in N} R_{i}$ is isomorphic to $\tilde{\Pi}_{i \in N} R$.

Proof. Define a mapping $f: \widetilde{\prod}_{i \in N} R \rightarrow \widetilde{\Pi}_{i \in N} R_{i}$ by $f\left(\left(x_{i}\right)_{i \in N}\right)=\left(x_{i} \bmod I_{i}\right)_{i \in N}$. This is obviously surjective. Assume that $f\left(\left(x_{i}\right)_{\imath \in N}\right)=0$. Then it follows by definition that for any $n \in \mathbb{N} x_{i} \in I_{i}+\mathfrak{m}_{i}^{n}$ for a.a. $i \in N$. Hence by the assumption $x_{i} \in \mathfrak{m}_{i}^{n}$ for a.a. $i \in N$. This shows $\left(x_{i}\right)_{i \in N}=0$.

(1.16) Corollary. Let $(R, \mathfrak{m})$ be a local ring and let $N=\mathbb{N}$. Then 
(i) $\tilde{\prod}_{i \in \mathbb{N}} R / \mathfrak{m}^{i} \cong \prod_{i \in \mathbb{N}} R$, and (ii) $\tilde{\Pi}_{i \in \mathbb{N}} R \cong \tilde{\Pi}_{i \in \mathbb{N}} \hat{R} \cong \tilde{\Pi}_{i \in \mathbb{N}} R^{h}$ where $\hat{R}$ (resp. $R^{h}$ ) denotes the completion (resp. henselization) of $R$.

Proof. (i) is immediate from (1.15). (ii) is the direct consequence of (i) and the fact; $R / \mathrm{m}^{i} \cong \hat{R} / \mathrm{m}^{i} \hat{R} \cong R^{h} / \mathrm{m}^{i} R^{h}$ for any $i \in \mathbb{N}$.

This result (1.16) (ii) will be generalized in (1.19).

Next we would like to prove an exactness criterion concerning separated ultraproducts. For this purpose we need some additional notation.

(1.17) Definition. Let $(R, \mathfrak{m})$ be a local ring and let $N \subset M$ be $R$ modules. Then we denote $a_{R}(N, M)=\inf \left\{r \in \mathbb{N} \mid \mathfrak{m}^{n} M \cap N=\mathfrak{m}^{n-r}\left(\mathfrak{m}^{r} M \cap N\right)\right.$ for all $n \geqq r\}$ and refer to it as the Artin-Rees number of $N \subset M$.

The following theorem is one of the main results of this section, and is quite useful in computing separated ultraproducts.

(1.18) Theorem (Exactness Theorem). Let $\left\{R_{i} \mid i \in N\right\}$ be a good family of local rings indexed by $N$ and let

$$
0 \longrightarrow M_{i}^{\prime} \underset{f_{i}}{\longrightarrow} M_{i} \underset{g_{i}}{\longrightarrow} M_{i}^{\prime \prime} \longrightarrow 0
$$

be an exact sequence of $R_{i}$-modules for $i \in N$. Assume that there is an integer $r$ such that $a_{R_{i}}\left(f_{i}\left(M_{i}^{\prime}\right),\left(M_{i}\right)\right) \leqq r$ for a.a. $i \in N$. Then one gets the exact sequence of $\tilde{\prod}_{i \in N} R_{i}$-modules;

$$
0 \longrightarrow \tilde{\prod}_{i \in N} M_{i}^{\prime} \longrightarrow \underset{f}{\longrightarrow} \tilde{\prod}_{i \in N} M_{i} \underset{\tilde{\theta}}{\longrightarrow} \tilde{\prod}_{i \in N} M_{i}^{\prime \prime} \longrightarrow 0
$$

Proof. Recall that $\tilde{f}\left(\left(x_{i}^{\prime}\right)_{i \in N}\right)=\left(f_{i}\left(x_{i}^{\prime}\right) \tilde{i}_{i \in N}\right.$ and $\tilde{g}\left(\left(x_{i}\right)_{\tilde{i} \in N}\right)=\left(g_{i}\left(x_{i}\right)\right)_{i \in N}$ for $\left(x_{i}^{\prime}\right)_{i \in N} \in \tilde{\Pi}_{i \in N} M_{i}^{\prime},\left(x_{i}\right)_{i \in N} \in \tilde{\Pi}_{i \in N} M_{i}$. Thus it is obvious that $\tilde{g} \cdot \tilde{f}=0$ and $\tilde{g}$ is surjective. We prove the injectivity of $\tilde{f}$. Assume that $\tilde{f}\left(\left(x_{i}^{\prime}\right)_{i \in N}\right)=0$ for $\left(x_{i}^{\prime}\right)_{i \in N} \in \tilde{\prod}_{i \in N} M_{i}^{\prime}$. Then it follows by definition that, for any integer $n$, it holds that $f_{i}\left(x_{i}^{\prime}\right) \in \mathfrak{m}_{i}^{n} M_{i}$ for a.a. $i \in N$. Hence if $n \geqq r$ then $f_{i}\left(x_{i}^{\prime}\right) \in$ $\mathfrak{m}_{i}^{n} M_{i} \cap f_{i}\left(M_{i}^{\prime}\right)=\mathfrak{m}_{i}^{n-r}\left(\mathfrak{m}_{i}^{r} M_{i} \cap f_{i}\left(M_{i}^{\prime}\right)\right) \subset \mathfrak{m}_{i}^{n-r} f_{i}\left(M_{i}^{\prime}\right)=f_{i}\left(\mathfrak{m}_{i}^{n-r} M_{i}^{\prime}\right)$ for those $i \in N$. Since $f_{i}$ is injective, it follows that $x_{i}^{\prime} \in \mathfrak{m}_{i}^{n-r} M_{i}^{\prime}$ for a.a. $i \in N$. Hence we have $\left(x_{i}^{\prime}\right)_{i \in N}=0$. This proves the injectivity of $\tilde{f}$.

Next we prove that $\operatorname{Ker}(\tilde{g}) \subset \tilde{f}\left(\tilde{\Pi}_{i \in N} M_{i}^{\prime}\right)$. For this, assume that $\tilde{g}\left(\left(x_{i}\right)_{i \in N}\right)=0\left(\left(x_{i}\right)_{i \in N} \in \widetilde{\prod}_{i \in N} M_{i}\right)$. Then we have that, for any integer $n$, $g_{i}\left(x_{i}\right) \in \mathfrak{m}_{i}^{n} M_{i}^{\prime \prime}$ for a.a. $i \in N$. This means that $x_{i} \in \mathfrak{m}_{i}^{n} M_{i}+f_{i}\left(M_{i}^{\prime}\right)$ for a.a. $i \in N$. Thus for any integer $n$, we can find elements $y_{i}(n) \in f_{i}\left(M_{i}^{\prime}\right)$ for a.a. $i \in N$ such that $x_{i}-y_{i}(n) \in \mathfrak{m}_{i}^{n} M_{i}$ for those $i \in N$. Note that this implies that $\left(x_{i}\right)_{i \in N}-\left(y_{i}(n)\right)_{i \in N} \in \tilde{\mathfrak{m}}^{n} \tilde{\prod}_{i \in N} M_{i}$ by (1.10). Also note that, for $m>n>r$, 
$y_{i}(m)-y_{i}(n) \in \mathfrak{m}_{i}^{n} M_{i} \cap f_{i}\left(M_{i}^{\prime}\right) \subset \mathfrak{m}_{i}^{n-r} f_{i}\left(M_{i}^{\prime}\right)$ for a.a. $i \in N$. Thus if we denote $y(n)=\left(y_{i}(n)\right)_{i \in N}$ then $\{y(n) \mid n \in \mathbb{N}\}$ forms a Cauchy sequence in $\tilde{f}\left(\tilde{\prod}_{i \in N} M_{i}^{\prime}\right)$. Note that $\tilde{f}\left(\tilde{\Pi}_{i \in N} M_{i}^{\prime}\right)$ is a complete module by (1.11) and by the injectivity of $\tilde{f}$. Thus there is an element $z$ of $\tilde{f}\left(\tilde{\prod}_{i \in N} M_{i}^{\prime}\right)$ to which $y(n)$ converges. Therefore, for any integer $m$, there is an integer $n$ satisfying $z-\left(x_{i}\right)_{i \in N}=$ $(z-y(n))-\left(\left(x_{i}\right)_{i \in N}-y(n)\right) \in \tilde{\mathfrak{m}}^{m} \tilde{\prod}_{i \in N} M_{i}$. Since $\tilde{\prod}_{i \in N} M_{i}$ is a separated module by (1.11), it follows that $\left(x_{i}\right)_{i \in N}=z \in \tilde{f}\left(\tilde{\prod}_{i \in N} M_{i}^{\prime}\right)$.

Remark that the Exactness Theorem (1.18) fails without the condition of the uniform finiteness of Artin-Rees numbers. See Example (1.25).

The following generalizes (1.6).

(1.19) Corollary. If $\left\{R_{i} \mid i \in N\right\}$ is a good family of local rings, then $\tilde{\prod}_{i \in N} R_{i} \cong \tilde{\prod}_{i \in N} \hat{R}_{i} \cong \tilde{\prod}_{i \in N} R_{i}^{h}$.

Proof. We only prove the first isomorphism. Note that $a_{R_{i}}\left(R_{i}, \hat{R}_{i}\right)=0$. Hence by the Exactness Theorem (1.18) we have the following exact sequence:

$$
0 \longrightarrow \tilde{\prod}_{i \in N} R_{i} \longrightarrow \tilde{\prod}_{i \in N} \hat{R}_{i} \longrightarrow \tilde{\prod}_{i \in N} \hat{R}_{i} / R_{i} \longrightarrow 0
$$

Remark that $\tilde{\Pi}_{i \in N} \hat{R}_{i}$ in this sequence is not a separated ultraproduct of $\hat{R}_{i}$ as $\hat{R}_{i}$-modules but as $R_{i}$-modules. However the both ones coincide with each other in this case since $\mathfrak{m}_{i} \hat{R}_{i}=\hat{\mathfrak{m}}_{i}$ for all $i \in N$. Thus it is sufficient to show that $\tilde{\prod}_{i \in N} \hat{R}_{i} / R_{i}=0$. This is, however, obvious from the fact that, for any $x_{i} \in \hat{R}_{i}$ and for any integer $n, x_{i} \in R_{i}+\mathfrak{m}_{i}^{n} \hat{R}_{i}$.

As a consequence of (1.19) we get the following example which generalizes [BDLD; Lemma (3.4) and Lemma (3.5)].

(1.20) ExAmple. Let $K_{\imath}$ be local rings with maximal ideal $\mathfrak{m}_{i}$ and $R_{i}=K_{i}\left[X_{1}, X_{2}, \cdots, X_{n}\right]_{\left(\mathrm{m}_{i}, X_{1}, X_{2}, \cdots, X_{n}\right)}$ (or $\left.R_{i}=K_{i}\left[\left[X_{1}, X_{2}, \cdots, X_{n}\right]\right]\right)$ for $i \in N$. Then we have

$$
\prod_{i \in N} R_{i} \cong \tilde{K}\left[\left[X_{1}, X_{2}, \cdots, X_{n}\right]\right]
$$

where $\tilde{K}$ denotes $\tilde{\Pi}_{i \in N} K_{i}$.

Proof. By virtue of (1.19) we may assume that $R_{i}=K_{i}\left[\left[X_{1}, X_{2}, \cdots, X_{n}\right]\right]$ where $K_{i}(i \in N)$ are all complete local rings. Define a mapping $f: \widetilde{\prod}_{i \in N} R_{i}$ $\rightarrow \tilde{K}\left[\left[X_{1}, X_{2}, \cdots, X_{n}\right]\right]$ by the following: For $\left(p_{i}(\underline{X})\right)_{i \in N}^{\sim} \in \tilde{\prod}_{i \in N} R_{i}$ where $p_{i}(\underline{X})=\sum_{m \in \mathbb{N}^{n}} a_{i}(m) \underline{X}^{m}\left(a_{i}(m) \in K_{i}\right)$, we set $f\left(\left(p_{i}(\underline{X})\right)_{i \in N}\right):=\sum_{m \in \mathbb{N} n}\left(a_{i}(m)\right)_{i \in N} \underline{X}^{m}$. 
Note that $f$ gives a well defined ring homomorphism.

For any element $q(\underline{X})=\sum_{m \in \mathbb{N}^{n}}\left(a_{i}(m)\right)_{i \in N} \underline{X}^{m} \in \tilde{K}[[X]], p:=\left(\sum_{m \in \mathbb{N}^{n}} a_{i}(m)\right.$ $\left.\cdot \underline{X}^{m}\right)_{i \in N} \in \tilde{\prod}_{i \in N} R_{i}$ satisfies that $f(p)=q(\underline{X})$, hence $f$ is surjective.

To prove the injectivity of $f$ assume that $f\left(\left(p_{i}(\underline{X})\right)_{i \in N}\right)=0$ for $p_{i}(\underline{X})$ $=\sum_{m \in \mathbb{N}^{n}} a_{i}(m) \underline{X}^{m} \in R_{i}$. Then by definition $\left(a_{i}(m)\right)_{i \in N}=0$ for any $m \in \mathbb{N}^{n}$, hence for any integer $k$ it holds that $a_{i}(m) \in \mathfrak{m}_{i}^{k}$ for $|m| \leqq k$ and for a.a. $i \in N$. This implies that $p_{i}(\underline{X}) \in\left(\mathfrak{m}_{i}, X_{1}, X_{2}, \cdots, X_{n}\right)^{k}$ for those $i \in N$, hence $\left(p_{i}(\underline{X})\right)_{i \in N}=0$.

(1.21) Notation. Let $R$ be a local ring. We call $\tilde{\prod}_{i \in N} R$ the separated ultrapower of $R$ on $N$ and denote it by $\tilde{R}^{N}$ (or simply $\tilde{R}$ if it causes no confusion). In the same way the separated ultrapower of $R$-module $M$ on $N$ is defined as $\tilde{\Pi}_{i \in N} M$ and is denoted by $\tilde{M}^{N}$ (or $\tilde{M}$ ).

Note that there is a natural ring homomorphism of $R$ to $\tilde{R}$ by placing an element of $R$ in a diagonal line, i.e. $x \mapsto(x)_{i \in N}$.

(1.22) Proposition. Let $R$ be a local ring. Assume that $\hat{R}=S / I$ where $S$ is a regular local ring and $I$ is an ideal of $S$. (Note that it is always possible to describe $\hat{R}$ in this manner by Cohen's theorem.) Then it holds that $\tilde{R}=\tilde{S} / I \tilde{S}$.

Here we should notice that $\tilde{S}$ is also a regular local ring by (1.14).

Proof. By virtue of (1.19) we may assume that $R=S / I$. In the exact sequence; $0 \rightarrow I \rightarrow S \rightarrow R \rightarrow 0$, it is trivial that $a_{S}(I, S)$ is constant for $i \in N$. Hence by the Exactness Theorem (1.18) we have an equality $\tilde{R}=\tilde{S} / \tilde{I}$. It remains to prove that $\tilde{I}=I \tilde{S}$. Note that $\tilde{I}$ is the ideal of $\tilde{S}$ consisting of those elements $\left(a_{i}\right)_{i \in N} \in \tilde{S}$ such that $a_{i} \in I$ for all $i \in N$. Thus it is obvious that $I \tilde{S} \subset \tilde{I}$. Assume that $\left(x_{i}\right)_{\tilde{i} \in N} \in \tilde{I}$. If $I$ is generated by $f(1), f(2), \cdots, f(k)$ then each $x_{i}$ can be written as $\sum_{j=1}^{n} a_{i}(j) f(j)$ for some $a_{i}(j) \in S$. Hence $\left(x_{i}\right)_{\tilde{i} \in N}=\sum_{j=1}^{n}\left(a_{i}(j)\right)_{i \in N}(f(j))_{i \in N} \in I \tilde{S}$, which proves $I \tilde{S} \supset \tilde{I}$.

(1.23) CoRollary. Let $R$ be a local ring such that $\hat{R}$ has a coefficient field $K$. Then there is an isomorphism of rings;

$$
\tilde{R} \cong \hat{R} \hat{\otimes}_{K} \tilde{K}
$$

where $\tilde{K}$ is the separated ultrapower of $K$ and $\hat{\otimes}_{K}$ denotes the complete tensor product over $K$.

Proof. By the assumption it can be written that $\hat{R}=K\left[\left[X_{1}, X_{2}, \cdots\right.\right.$, 
$\left.\left.X_{n}\right]\right] / I$ for some integer $n$ and some ideal $I$ of $K\left[\left[X_{1}, X_{2}, \cdots, X_{n}\right]\right]$. Then by (1.22) and (1.20) we see that

$$
\begin{aligned}
\tilde{R} & \cong \hat{\hat{R}} \cong K\left[\left[X_{1}, X_{2}, \cdots, X_{n}\right]\right]^{\sim} / I K\left[\left[X_{1}, X_{2}, \cdots, X_{n}\right]\right]^{\sim} \\
& \cong \tilde{K}\left[\left[X_{1}, X_{2}, \cdots, X_{n}\right]\right] / I \tilde{K}\left[\left[X_{1}, X_{2}, \cdots, X_{n}\right]\right] \\
& \cong \hat{R} \hat{\otimes}_{K} \tilde{K} .
\end{aligned}
$$

This corollary shows that $\tilde{R}$ is a faithfully flat $R$-module under the assumption that $R$ contains a field. We next prove this fact in general.

(1.24) Theorem. If $R$ is a local ring, then $\tilde{R}$ is a faithfully flat $R$ algebra.

Proof. Notice that if $0 \rightarrow M^{\prime} \rightarrow M \rightarrow M^{\prime \prime} \rightarrow 0$ is an exact sequence of finitely generated $R$-modules, then $0 \rightarrow \tilde{M}^{\prime} \rightarrow \tilde{M} \rightarrow \tilde{M}^{\prime \prime} \rightarrow 0$ is an exact sequence of $\tilde{R}$-modules. In fact since $M$ is finitely generated it follows by Artin-Rees lemma that $a_{R}\left(M^{\prime}, M\right)$ is finite, hence the Exactness Theorem (1.18) gives the exactness of the sequence; $0 \rightarrow \tilde{M}^{\prime} \rightarrow \tilde{M} \rightarrow \tilde{M}^{\prime \prime} \rightarrow 0$. This shows that the operation $\sim$ is an exact additive functor from the category of finitely generated $R$-modules to the category of $\tilde{R}$-modules. Since it trivially holds that $\tilde{M} \cong M \otimes_{R} \tilde{R}$ for a finitely generated free $R$-module $M$, the usual argument on functors shows that there is a natural isomorphism $\tilde{M} \cong M \otimes_{R} \tilde{R}$ for any finitely generated $R$-module $M$. This concludes that $\tilde{R}$ is flat over $R$.

We close this section by giving an example.

(1.25) Example. Let $R_{i}=K_{i}[[X, Y]] /\left(X^{2}+Y^{i}\right)$ for $i \in \mathbb{N}$ where $K_{i}$ are fields. Then we have

$$
\prod_{i \in \mathbb{N}} R_{i} \cong \tilde{K}[[X, Y]] /\left(X^{2}\right)
$$

where $\tilde{K}$ denotes $\tilde{\prod}_{i \in N} K_{i}$. To prove this fact we set $S_{\imath}=K_{i}[[X, Y]]$, $\tilde{S}=\tilde{\prod}_{i \in \mathbb{N}} S_{i}=\tilde{K}[[X, Y]]$ and $I_{i}=\left(X^{2}+Y^{i}\right) S_{i}$. It is easily observed that $a_{S_{i}}\left(I_{i}, S_{i}\right)=2$ for all $i \in \mathbb{N}$. Hence it follows by the Exactness Theorem (1.18) that $\tilde{\prod}_{i \in \mathbb{N}} R_{i} \cong \tilde{S} / \tilde{I}$ where $\tilde{I}$ is generated by a single element $\left(X^{2}+Y^{i}\right)_{i \in \mathbb{N}} \in \tilde{S}$. Since, for any $n \in \mathbb{N}, Y^{i} \in \mathfrak{m}_{i}^{n}$ for a.a. $i \in \mathbb{N}$, we know that $\left(Y^{i}\right)_{i \in \mathbb{N}}=0$. Hence $\tilde{I}$ is generated by $X^{2} \in \tilde{S}$.

This example shows that the Exactness Theorem (1.18) fails when there is no bound on Artin-Rees numbers. For instance consider an exact 
sequence;

$$
0 \longrightarrow R_{i} \underset{X}{\longrightarrow} R_{i} \longrightarrow R_{i} / X R_{i} \longrightarrow 0
$$

for all $i \in \mathbb{N}$. In this case the following sequence is not exact:

$$
0 \longrightarrow \tilde{\prod}_{i \in \mathbb{N}} R_{i} \longrightarrow \tilde{\prod}_{i \in \mathbb{N}} R_{i} \longrightarrow \tilde{\prod}_{i \in \mathbb{N}} R_{i} / X R_{i} \longrightarrow 0
$$

Note that $a_{R_{i}}\left(X R_{i}, R_{i}\right)=i-1$ for all $i \in \mathbb{N}$.

\section{§ 2. Rich algebras and poor algebras over Artinian local rings}

In this section we mainly concentrate our attention on the following

(2.1) Problem. Let $T$ be a Noetherian ring and let $R$ be a $T$-algebra. Under what condition does there exist a non-trivial $R$-module which is injective as a $T$-module? (To simplify the notation we shall call such a module a $T$-injective $R$-module. Note that under this name we only consider non-trivial ones.)

The problem is reduced to the case that $T$ is an Artinian local ring by the following

(2.2) Lemma. Let $T$ and $R$ be as above. Then the following conditions are equivalent.

(i) There exists a T-injective R-module.

(ii) There is a minimal prime ideal $\mathfrak{p}$ of $T$ such that there exists a $T_{\mathrm{p}}$-injective $R_{\mathrm{p}}$-module.

Before proceeding to the proof we note the following fact: Let $A$ be a local ring with maximal ideal $m$ and let $E$ be the injective envelope of $A / \mathfrak{m}$ over $A$. For an $A$-module $M, M$ is a flat (resp. injective, non-trivial) $A$-module if and only if $\operatorname{Hom}_{A}(M, E)$ is an injective (resp. flat, non-trivial) $A$-module.

Proof of (2.2). (ii) $\Rightarrow$ (i). If $M$ is a $T_{p}$-injective $R_{\mathrm{p}}$-module, then $M$ itself is an $R$-module and is an injective $T$-module.

(i) $\Rightarrow$ (ii). Assume that $M$ is a $T$-injective $R$-module. Let $\mathfrak{q}$ be a prime ideal of $T$ such that $\mathfrak{q}$ has the minimum height among $\operatorname{Ass}_{T}(M)$. Since $M$ is $T$-injective, we see that $M_{a}$ is a direct sum of copies of $E_{T_{q}}\left((T / \mathfrak{q})_{q}\right)$ as $T_{q}$-module. (See $[M]$.) Denote $N:=\operatorname{Hom}_{T_{q}}\left(M_{q}, E_{T_{q}}\left((T / \mathfrak{q})_{q}\right)\right)$. Then it is easily verified that $N$ is a direct product of copies of $\left(T_{q}\right)^{\wedge}$, hence is flat over $T_{\mathfrak{q}}$. If we take a minimal prime ideal $\mathfrak{p}$ of $T$ contained in 
$\mathfrak{q}$, then we see that $N_{\mathfrak{p}}$ is a non-trivial $T_{\mathfrak{p}}$-flat $R_{\mathfrak{p}}$-module. It follows from the above remark that $\operatorname{Hom}_{T \mathfrak{p}}\left(N_{\mathfrak{p}}, E_{T \downarrow}\left((T / \mathfrak{p})_{\mathfrak{p}}\right)\right)$ is a $T_{\mathfrak{p}}$-injective $R_{\mathfrak{p}}$-module.

(2.3) Remark. By the fact remarked before the above proof one sees that if $T$ is a local ring, then there is a $T$-injective $R$-module if and only if there is a $T$-flat $R$-module.

By virtue of (2.2) we may restrict ourselves to consider the case that $T$ is an Artinian local ring. Thus in the rest of this section $T$ always denotes an Artinian local ring with maximal ideal $\mathfrak{m}$ and $k=T / \mathrm{m}$. We make the following definition in this case.

(2.4) Definition. We say that a $T$-algebra $R$ is a rich T-algebra if there is a $T$-injective $R$-module, otherwise we call it a poor $T$-algebra.

We exhibit some formal results in the following.

(2.5) LEMma. (a) Consider the following commutative diagram of rings;<smiles>[R20]C=[Y2]1[Y][Tl][Y]1[Y]</smiles>

where $T$ and $T^{\prime}$ are Artinian local rings.

(a-1) If $R$ is a rich T-algebra, then $R^{\prime}$ is a rich $T^{\prime}$-algebra.

(a-2) If $T^{\prime}$ is flat over $T$, then the converse of (a-1) is also valid.

(b) Consider ring homomorphisms $T \rightarrow R \rightarrow S$.

(b-1) If $S$ is a rich T-algebra, then $R$ is also a rich T-algebra.

(b-2) Assume that $R$ is also an Artinian local ring. If $S$ is a rich $R$-algebra and if $R$ is a rich T-algebra, then $S$ is a rich T-algebra.

Proof. (a-1) Let $M$ be a $T$-injective $R$-module. Setting

$$
M^{\prime}=\operatorname{Hom}_{T}\left(T^{\prime}, M\right),
$$

we see that $M^{\prime}$ is an $R^{\prime}$-module by the action; $((r \otimes t) f)\left(t^{\prime}\right)=r f\left(t t^{\prime}\right)$ for $r \otimes t \in R^{\prime}=R \otimes T^{\prime}, t^{\prime} \in T^{\prime}$ and $f \in M^{\prime}$. It is well known that $M^{\prime}$ is an injective module as a $T^{\prime}$-module. Since $T$ is Artinian local and $M$ is injective, we have $M^{\prime} \neq 0$.

(a-2) If $R^{\prime}$ is a rich $T^{\prime}$-algebra, then by (2.2) there is a $T^{\prime}$-flat $R^{\prime}$-module $M^{\prime}$. Since $T^{\prime}$ is a flat $T$-algebra, $M^{\prime}$ is also flat over $T$. Hence by (2.2) we see that $R$ is a rich $T$-algebra.

(b-1) Trivial. 
(b-2) Let $M$ be an $R$-injective $S$-module and let $N$ be a $T$-flat $R$-module. If we denote $L=\operatorname{Hom}_{R}(N, M)$, then $L$ is obviously an $S$-module by the action; $(r f)(n)=r(f(n))$ for $r \in S, n \in N$ and $f \in L$. Note that there is an isomorphism of functors; $\operatorname{Hom}_{T}(, L) \cong \operatorname{Hom}_{R}\left(\otimes_{T} N, M\right)$ and hence this is an exact functor. This shows that $L$ is an injective module as a $T$ module.

We next give some information about ring theoretic properties of rich alegbras.

(2.6) Proposition. (i) If a ring homomorphism $T \rightarrow R$ has a ring retraction, then $R$ is a rich T-algebra.

(ii) If $R$ is a flat T-algebra, then $R$ is a rich T-algebra.

(iii) If $R$ is a rich T-algebra, then $T$ is a direct summand of $R$ as a $T$-module. In particular, the natural ring homomorphism $T \rightarrow R$ is pure.

Proof. (i) is clear from the fact that, in this case, every $T$-module is naturally an $R$-module.

(ii) is immediate from (2.3).

(iii) Let $M$ be a $T$-injective $R$-module. We know by [M] that $M$ is a direct sum of copies of $E$ where $E$ denotes the injective envelope of $k$ as a $T$-module. In particular there is an embedding $E \rightarrow M$ as a $T$-module. Taking the dual of it we have a map;

$$
f: \operatorname{Hom}_{T}(M, E) \rightarrow \operatorname{Hom}_{T}(E, E) \cong T .
$$

(See [M] for the last equality.) Since $f$ is surjective, there is an element $x$ of $\operatorname{Hom}_{T}(M, E)$ such that $f(x)=1$. We define an $R$-module homomorphism $g: R \rightarrow \operatorname{Hom}_{T}(M, E)$ by sending 1 to $x$ and define $h:=f \cdot g$. It is easily seen that $h$ is a $T$-module homomorphism of $R$ to $T$ satisfying $h(1)=1$. This proves (iii).

In general the converse of this proposition fails.

(2.7) ExAmple. (i) Let $k$ be a field and let $T=k[[x, w]] /\left(x^{2}, w^{4}\right)$ and $R=k[[x, y, z, w]] /\left(x^{2}, w^{4}, x w-y z, x^{2} z-y^{3}, y w^{2}-z^{3}, x z^{2}-y^{2} w\right)$ where $T$ is naturally a subring of $R$. One can show that $T \rightarrow R$ has no ring retraction and $R$ is not flat over $T$. However we will see later that $R$ is indeed a rich $T$-algebra. This will be immediate from Theorem (3.6).

(ii) Let $V$ be a discrete valuation ring with a prime element $t$ and let $T=V / t^{2} V$ and $R=T[X] /\left(t X, X^{2}+t\right)$. One can easily verify that $T$ is naturally a subring of $R$. Since $T$ is a Gorenstein ring of dimension $0, T$ 
is a direct summand of $R$ as a $T$-module. It will be proved in (2.11) that $R$ is a poor $T$-algebra.

One of the purposes of this section is to construct the generic poor algebras. We first give a concrete description of those algebras, and afterward we will verify that they satisfy the necessary properties to be generic.

(2.8) Definition of $u_{l}(T)$. Let $(T, \mathfrak{m}, k)$ be an Artinian local ring as above. Denote a minimal free resolution of $k$ as a $T$-module by the following;

$$
\begin{gathered}
\longrightarrow \longrightarrow T^{d^{\prime}} \longrightarrow T^{d} \longrightarrow\left[\begin{array}{c}
x_{1} \\
\vdots \\
\vdots \\
x_{d}
\end{array}\right] \\
\left.\longrightarrow a_{i j}\right] \quad \boldsymbol{x}=0
\end{gathered}
$$

where $A$ is a $d^{\prime} \times d$-matrix whose components are in $T$ and $x_{i} \in \mathfrak{m}$. Notice that both $d$ and $d^{\prime}$ are uniquely determined by $T$. We prepare a number of variables over $T$. For any integer $n$ and $j(1 \leqq j \leqq n)$, consider the following matrices;

$$
\begin{aligned}
& \boldsymbol{Y}_{n}=\left[\begin{array}{ccccc}
y_{11}^{(n)} \cdots y_{1 n}^{(n)} & 0 & 0 & \cdots \\
\vdots & \vdots & \vdots & \vdots & \\
y_{d 1}^{(n)} \cdots & y_{d n}^{(n)} & 0 & 0 & \cdots
\end{array}\right]=d \times \infty \text {-matrix } \\
& Z_{n j}=\left[\begin{array}{ccc}
z_{11}^{(n j)} \cdots & z_{1 d}^{(n j)} \\
\vdots & & \vdots \\
z_{d^{\prime} 1}^{(n j)} & \cdots & z_{d^{\prime} d}^{(n j)}
\end{array}\right]=d^{\prime} \times d \text {-matrix, and } \\
& W_{n}=\left[w_{1}^{(n)}, \cdots, w_{d}^{(n)}\right]=1 \times d \text {-matrix, }
\end{aligned}
$$

where all $y_{i j}^{(n)}, \boldsymbol{z}_{k l}^{(n j)}$ and $w_{i}^{(n)}$ are indeterminates over $T$. We also denote

$$
\left(\boldsymbol{Y}_{n}, \boldsymbol{x}\right)=\left[\begin{array}{cccccc}
y_{11}^{(n)} & \cdots & y_{1 n}^{(n)} & x_{1} & 0 & \cdots \\
\vdots & & \vdots & \vdots & \vdots & \\
y_{d 1}^{(n)} & \cdots & y_{d n}^{(n)} & x_{d} & 0 & \cdots
\end{array}\right]=d \times \infty \text {-matrix }
$$

and $\boldsymbol{E}=[1,0,0, \cdots]=1 \times \infty$-matrix. Under these notation we define the $T$-algebra $u_{l}(T)$ for an integer $l$ as follows:

$$
u_{l}(T):=\frac{T\left[\boldsymbol{Y}_{1}, \boldsymbol{Y}_{2}, \cdots, \boldsymbol{Y}_{l}, \boldsymbol{Z}_{n j}(1 \leqq n \leqq l-1,1 \leqq j \leqq n), W_{1}, W_{2}, \cdots, \boldsymbol{W}_{l}\right]}{\left(A \boldsymbol{Y}_{1}, A \boldsymbol{Y}_{n+1}-\sum_{j=1}^{n} \boldsymbol{Z}_{n j}\left(\boldsymbol{Y}_{j} \boldsymbol{x}\right)(1 \leqq n \leqq l-1), \boldsymbol{E}-\sum_{j=1}^{l} \boldsymbol{W}_{j}\left(\boldsymbol{Y}_{j}, \boldsymbol{x}\right)\right)}
$$

where $T\left[\boldsymbol{Y}_{1}, \boldsymbol{Y}_{2}, \cdots, \boldsymbol{Y}_{l}, \boldsymbol{Z}_{n j}(1 \leqq n \leqq l-1,1 \leqq j \leqq n), W_{1}, \boldsymbol{W}_{2}, \cdots, \boldsymbol{W}_{l}\right]$ denotes 
the polynomial ring over $T$ with the variables which appear in those matrices $\boldsymbol{Y}_{1}, \boldsymbol{Y}_{2}, \cdots, \boldsymbol{Y}_{n}, \boldsymbol{Z}_{n j}(1 \leqq n \leqq l-1,1 \leqq j \leqq n)$ and $W_{1}, W_{2}, \cdots, W_{l}$, and the ideal described in the denominator expresses the ideal generated by the elements which appear in those matrices $A Y_{1}, \cdots$, etc.

It can be easily verified that there is a natural isomorphism;

$$
u_{l}(T) /\left(Y_{l}, Z_{l-1, j}(1 \leqq j \leqq l-1), W_{l}\right) u_{l}(T) \cong u_{l-1}(T),
$$

hence there is a sequence of surjective $T$-algebra maps;

$$
\cdots \longrightarrow u_{l}(T) \longrightarrow u_{l_{-1}}(T) \longrightarrow \cdots \longrightarrow u_{2}(T) \longrightarrow u_{1}(T) .
$$

(2.9) Remark. The $T$-algebra $u_{l}(T)$ does not depend on the choice of minimal free resolution of $k$. In fact if

$$
\cdots \longrightarrow T^{d^{\prime}} \underset{\boldsymbol{B}}{\longrightarrow} T^{d} \underset{\boldsymbol{y}}{\longrightarrow} T \longrightarrow k \longrightarrow 0
$$

is another minimal free resolution, then it is observed that there are invertible matrices $C$ and $D$ which satisfy $A C=D B$ and $C y=x$. Then by the change of variables; $\boldsymbol{Y}_{n} \mapsto C^{-1} \boldsymbol{Y}_{n}, Z_{n j} \mapsto D^{-1} Z_{n j} C, W_{i} \mapsto W_{i} C$, we easily see that both $T$-algebras constructed from $(\boldsymbol{A}, \boldsymbol{x})$ and $(\boldsymbol{B}, \boldsymbol{y})$ are isomorphic to each other.

One of the main results of this section is the following

(2.10) Theorem (Existence of generic poor algebras). A T-algebra $R$ is a poor T-algebra if and only if it contains a specialization of $u_{l}(T)$ as a $T$-algebra for some integer $l$.

Before proceeding to the proof of this theorem we give some examples.

(2.11) Example. (i) Under the notation as in (2.8),

$$
u_{1}(T)=T\left[Y_{1}, W_{1}\right] /\left(A Y_{1}, E-W_{1}\left(Y_{1}, \boldsymbol{x}\right)\right)
$$

where

$$
Y_{1}=\left[\begin{array}{cccc}
y_{1} & 0 & 0 & \cdots \\
\vdots & \vdots & \vdots & \\
y_{d} & 0 & 0 & \cdots
\end{array}\right]
$$

and $W_{1}=\left[w_{1}, w_{2}, \cdots, w_{d}\right]$. Since $A$ contains the Koszul relation of $\boldsymbol{x}$, it holds that $x_{i} y_{j}=x_{j} y_{\imath}(1 \leqq i, j \leqq d)$ in $u_{1}(T)$. Therefore $x_{i}=x_{i} \sum_{j=1}^{d} w_{j} y_{j}$ $=\sum_{j=1}^{d} w_{j} x_{j} y_{i}=0$ in $u_{1}(T)$. Thus we conclude that 


$$
u_{1}(T)=u_{1}(T) / \mathfrak{m} u_{1}(T) \cong k\left[y_{1}, y_{2}, \cdots, y_{d}, w_{1}, w_{2}, \cdots, w_{d}\right] /\left(1-\sum_{i} y_{i} w_{i}\right)
$$

(ii) Let $V$ be a discrete valuation ring with a prime element $t$ and let $T=V / t^{2} V$. In this case

$$
\cdots \longrightarrow T \underset{t}{\longrightarrow} T \underset{t}{\longrightarrow} T \longrightarrow 0
$$

gives a minimal free resolution of $k$. (i.e. $A=(t)$ and $x=t$ in (2.8).) Hence we have

$$
\begin{aligned}
u_{1}(T) \cong k[y, w] /(1-y w) \cong k\left[y, y^{-1}\right] \\
u_{2}(T)=T\left[Y_{1}, Y_{2}, Z_{11}, W_{1}, W_{2}\right] /\left(t Y_{1}, t Y_{2}-Z_{11}\left(Y_{1}, t\right),\right. \\
\left.E-W_{1}\left(Y_{1}, t\right)-W_{2}\left(Y_{2}, t\right)\right),
\end{aligned}
$$

and

$$
u_{3}(T)=T\left[Y_{1}, Y_{2}, Y_{3}, Z_{11}, Z_{21}, Z_{22}, W_{1}, W_{2}, W_{3}\right] / I
$$

where $I$ is generated by $t \boldsymbol{Y}_{1}, t \boldsymbol{Y}_{2}-Z_{11}\left(\boldsymbol{Y}_{1}, t\right), t \boldsymbol{Y}_{3}-Z_{21}\left(\boldsymbol{Y}_{1}, t\right)-Z_{22}\left(\boldsymbol{Y}_{2}, t\right)$ and $E-W_{1}\left(Y_{1}, t\right)-W_{2}\left(Y_{2}, t\right)-W_{3}\left(Y_{3}, t\right)$.

If we denote $R=T[X] /\left(t X, X^{2}+t\right)$ as in (2.7), then we see that $R$ is obtained from $u_{3}(T)$ by the following specialization;

$$
\begin{aligned}
& Y_{1} \longmapsto(-X, 0,0, \cdots), Y_{2} \longmapsto(1,0,0, \cdots), Y_{3} \longmapsto(0,0, X, 0, \cdots) \\
& Z_{11} \longmapsto(X), Z_{21} \longmapsto(0), Z_{22} \longmapsto(0), \\
& W_{1} \longmapsto(0), W_{2} \longmapsto(1), W_{3} \longmapsto(X) .
\end{aligned}
$$

Hence Theorem (2.10) asserts that $R$ is a poor $T$-algebra. (See (2.7).)

Roughly speaking, the proof of Theorem (2.10) is done in the following course: First we construct a universal $R$-module which is possibly $T$ injective if it is non-trivial. One can see that this construction is the same one as in the proof of the existence of injective envelopes in [CE; Theorem (3.3)]. Next we verify that the constructed module is non-trivial if and only if $R$ contains a specialization of some $u_{l}(T)$. The reader will notice that, on the whole, our method is similar to the one used in $\left[\mathrm{H}_{1}\right.$; $\S 4]$.

For this purpose we begin with the following

(2.12) Definition. Let $(T, \mathfrak{m}, k)$ be an Artinian local ring as above and let $R$ be a $T$-algebra. For an $R$-module $L$ and a $T$-homomorphism $f$ of $m$ to $L$, we define 


$$
L^{(1)}=L \oplus R / \sum_{z \in \mathfrak{m}}(f(z), z) R
$$

and call $L^{(1)}$ the first modification of $L$ with respect to $f$. Note that $L^{(1)}$ is an $R$-module and there is a natural $R$-homomorphism of $L$ to $L^{(1)}$. In general, we may have a sequence

$$
L \longrightarrow L^{(1)} \longrightarrow L^{(2)} \longrightarrow \cdots \longrightarrow L^{(r)}
$$

in which $L^{(i+1)}$ is the first modification of $L^{(i)}$ with respect to some $f_{i} \in$ $\operatorname{Hom}_{T}\left(\mathrm{~m}, L^{(i)}\right)$. Then we say that $L^{(r)}$ is an $(r$-th) modification of $L$. Denote by $\mathfrak{M}(R)$ the set of all the modifications of $R$. We can prove that $\mathfrak{M}(R)$ forms an inductive system of $R$-modules by those natural homomorphisms. In fact to prove this it suffices to see the following: If $L_{f}^{(1)}$ and $L_{g}^{(1)}$ are the first modifications of $L$ with respect to $f$ and $g$ respectively, then there is a modification $L^{\prime}$ of $L$ which is also a modification of both $L_{f}^{(1)}$ and $L_{g}^{(1)}$ such that the diagram of natural mappings;<smiles>[14CH][14CH][Tl]</smiles>

is commutative. For this it is sufficient to put

$$
L^{\prime}=L \oplus R \oplus R / \sum_{z \in \mathrm{m}}(f(z), z, 0) R+(g(z), 0, z) R .
$$

Thus we may define the inductive limit of elements in $\mathfrak{M}(R)$ :

$$
M(R / T):=\lim _{L \overline{\in M(R)}} L .
$$

Note that $M(R / T)$ is an $R$-module and there is a natural $R$-homomorphism of $R$ to $M(R / T)$.

(2.13) Lemma. $\operatorname{Ext}_{T}^{1}(k, M(R / T))=0$.

Proof. We have to prove that any $T$-homomorphism $f: \mathfrak{m} \rightarrow M(R / T)$ extends to a $T$-homomorphism $g: T \rightarrow M(R / T)$. Since $\mathrm{m}$ is finitely generated, there is an $l$-th modification $L$ of $R$ for some integer $l$ and a $T$-homomorphism $f_{L}: \mathfrak{m} \rightarrow L$ such that the following is commutative

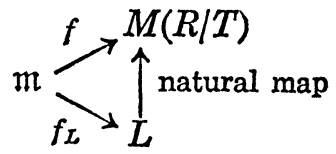


Let $L^{(1)}$ be the first modification of $L$ with respect to $f_{L}$. Then define a $T$-homomorphism $h: T \rightarrow L^{(1)}=L \oplus R / \sum_{z \in \mathrm{m}}\left(f_{L}(z), z\right) R$ by $h(x):=$ the image of $(0,-x) \in L \oplus R$ in $L^{(1)}$ for $x \in T$. We easily see that the diagram;

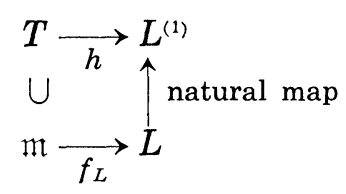

is commutative. If $g$ denotes the map of $T$ to $M(R / T)$ induced by $h$, then it is obvious that $\left.g\right|_{\mathrm{m}}=f$ as desired.

(2.14) Corollary. If $M(R / T) \neq 0$, then $M(R / T)$ is a T-injective $R$ module. In particular $R$ is a rich T-algebra in this case.

Proof. It is sufficient to notice the following fact: For any $T$-module $M$ it is $T$-injective if and only if $\operatorname{Ext}_{T}^{1}(k, T)=0$. (Since $T$ is Artinian, see [B; § 2]).

(2.15) Proposition. For a T-algebra $R$ the following conditions are equivalent.

(i) $R$ is a rich T-algebra.

(ii) $M(R / T) \neq 0$.

(iii) For any modification $L$ of $R$ and a natural map $r: R \rightarrow L$, it holds that $r(1) \neq 0$.

Proof. (iii) $\Rightarrow$ (ii). Trivial.

(ii) $\Rightarrow$ (i) was done in (2.14).

(i) $\Rightarrow$ (iii). Let $M$ be a $T$-injective $R$-module and let

$$
R \stackrel{r_{1}}{\longrightarrow} R^{(1)} \stackrel{r_{2}}{\longrightarrow} R^{(2)} \stackrel{r_{3}}{\longrightarrow} R^{(3)} \longrightarrow \cdots \stackrel{r_{l-1}}{\longrightarrow} R^{(l-1)} \stackrel{r_{l}}{\longrightarrow} R^{(l)}
$$

be a sequence of first modifications such that $L=R^{(l)}$. It suffices to prove the following

ClaIm. For any $R$-homomorphism $g: R \rightarrow M$ there is an $R$-homomorphism $h_{l}: L \rightarrow M$ which makes the following diagram commutative.

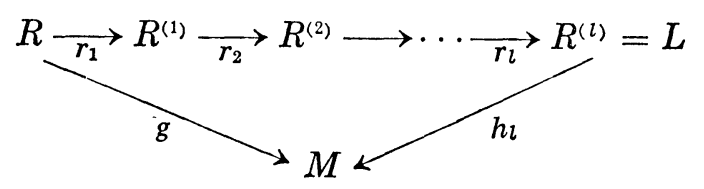

We prove this claim by induction on $l$. So assume that we already have an $R$-homomorphism $h_{l-1}: R^{(l-1)} \rightarrow M$ such that the diagram; 


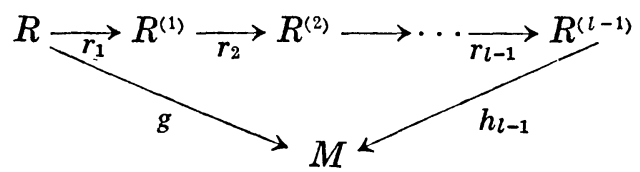

is commutative. We have only to prove the existence of an $R$-homomorphism $h_{l}: R^{(l)} \rightarrow M$ which makes the diagram;

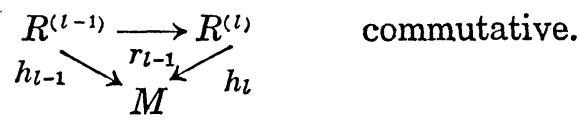

Now assume that $R^{(l)}$ is the first modification of $R^{(l-1)}$ with respect to $f \in \operatorname{Hom}_{T}\left(\mathfrak{m}, R^{(l-1)}\right)$, i.e. $R^{(l)}=R^{(l-1)} \oplus R / \sum_{z \in \mathfrak{m}}(f(z), z) R$. Since $M$ is a $T$-injective module, $h_{l-1} \cdot f$ extends to a $T$-homomorphism $h^{\prime}: T \rightarrow M$. We define the $R$-homomorphism $H: R^{(l-1)} \oplus R \rightarrow M$ by $H(y, r)=h_{l-1}(y)-r h^{\prime}(1)$. Then it is observed that $H(f(z), z)=0$ for any $z \in \mathfrak{m}$, and $H(y, 0)=h_{l-1}(y)$ for any $y \in R^{(l-1)}$. This shows that $H$ induces the $R$-homomorphism $h_{l}: R^{(l)}$ $\rightarrow M$ and the following diagram is commutative:

$$
\stackrel{R_{l-1}^{(l-1)}}{\stackrel{r_{l-1}}{\longrightarrow} R^{(l)}}
$$

This completes the proof of the proposition.

(2.16) Definition And Corollary, Let $R$ be a T-algebra and let $l$ be an integer. $R$ is said to be an l-poor T-algebra if there is an l-th modifcation $f_{l}: R \rightarrow R^{(l)}$ of $R$ such that $f_{l}(1)=0$. It trivially holds that

$\{1$-poor T-algebras $\} \subset\{2$-poor T-algebras $\} \subset \ldots$

$$
\subset\{l \text {-poor T-algebras }\} \subset\{(l+1) \text {-poor T-algebras }\} \subset \ldots
$$

and by (2.15) we see that

$$
\{\text { poor T-algebras }\}=\bigcup_{l=1}^{\infty}\{l \text {-poor T-algebras }\} \text {. }
$$

By virtue of (2.16), in order to prove Theorem (2.10), it suffices to show the following

(2.17) Propositron. $R$ is an l-poor T-algebra if and only if $R$ contains a specialization of $u_{l}(T)$.

For the proof of this proposition we need a lemma which is almost trivial. 
(2.18) Lemma. Assume that a minimal free resolution of $k$ is given in the following;

$$
\begin{aligned}
& T^{d^{\prime}} \longrightarrow T^{d} \longrightarrow T \longrightarrow k \longrightarrow 0 . \\
& \boldsymbol{A}=\left[a_{i j}\right] \quad \boldsymbol{x}=\left[\begin{array}{c}
x_{1} \\
\vdots \\
x_{d}
\end{array}\right]
\end{aligned}
$$

Then, for a T-module $L$, giving a T-homomorphism of $\mathfrak{m}$ to $L$ is equivalent to giving a sequence $\left(y_{1}, y_{2}, \cdots, y_{d}\right) \in L^{d}$ satisfying $\sum_{j=1}^{d} a_{i j} y_{j}=0$.

Proof. It is trivial by putting $y_{i}=f\left(x_{i}\right) \quad(i=1,2, \cdots, d)$ for $f \in$ $\operatorname{Hom}_{T}(\mathfrak{m}, L)$.

Proof of Proposition (2.17). For an $R$-module $L$ we know by (2.18) that any first modification $L^{(1)}$ of $L$ is described as follows;

$$
L^{(1)}=L \oplus R / \sum_{i=1}^{d}\left(y_{i}, x_{i}\right) R
$$

where $y_{i} \in L(i=1,2, \cdots, d)$ such that $A\left[\begin{array}{c}y_{1} \\ \vdots \\ y_{g}\end{array}\right]=0$ in $L . \quad$ Thus if $R \rightarrow R^{(1)} \rightarrow$ $R^{(2)} \rightarrow \cdots \rightarrow R^{(l)}$ is an $l$-th modification of $R$, then $R^{(l)}$ is given in the form;

$$
R^{l} /\left\{\left(y_{i 1}^{(j)}, y_{i 2}^{(j)}, \cdots, y_{i j}^{(j)}, x_{i}, 0, \cdots, 0\right) \mid 1 \leqq i \leqq d, 1 \leqq j \leqq l-1\right\} R
$$

where for any $j(1 \leqq j \leqq l-1)$ each row of the matrix $A \cdot\left[\begin{array}{ccc}y_{11}^{(j)} \cdots & y_{1 j}^{(j)} \\ \vdots & & \vdots \\ y_{d 1}^{(j)} & \cdots & y_{d j}^{(j)}\end{array}\right]$ is an $R$-linear combination of

$$
\left\{\left(y_{s 1}^{(k)}, y_{s 2}^{(k)}, \cdots, y_{s k}^{(k)}, x_{s}, 0, \cdots, 0\right) \mid 1 \leqq k \leqq j-1,1 \leqq s \leqq d\right\}
$$

Moreover the condition that 1 goes to 0 under the natural map of $R$ to $R^{(l)}$ is equivalent to that the vector $(1,0,0, \cdots, 0)$ with $l$ components is an $R$-linear combination of

$$
\left\{\left(y_{i 1}^{(j)}, y_{i 2}^{(j)}, \cdots, y_{i j}^{(j)}, x_{i}, 0, \cdots, 0\right) \mid 1 \leqq i \leqq d, 1 \leqq j \leqq l-1\right\}
$$

Putting together these informations we verify that $R$ is an $l$-poor $T$-algebra if and only if $R$ contains a solution of the following system of equations; 


$$
\begin{aligned}
A \boldsymbol{Y}_{n+1} & =\sum_{j=1}^{n} \boldsymbol{Z}_{n j}\left(\boldsymbol{Y}_{j}, \boldsymbol{x}\right) \quad(n=0,1, \cdots, l-1) \\
\boldsymbol{E} & =\sum_{i=1}^{l} \boldsymbol{W}_{i}\left(\boldsymbol{Y}_{i}, \boldsymbol{x}\right),
\end{aligned}
$$

where the notation is the same as in (2.8). This establishes (2.17), hence Theorem (2.10).

We may expect by Theorem (2.10) that the peculiarity of the structure of poor algebras comes from the property of generic ones. For instance we can prove the following proposition.

(2.19) Proposition. Let $R$ be a l-poor T-algebra. If $I$ is an ideal of $R$ which annihilates $\operatorname{Tor}_{1}^{T}(k, R)$, then $I^{l}=0$. In particular, $\mathfrak{m}^{l} R=0$.

Proof. By the assumption there is a $T$-algebra map of $u_{l}(T)$ to $R$. We denote the images of $\boldsymbol{Y}_{i}, Z_{i j}, W_{i}$ in $R$ by the same letters $\boldsymbol{Y}_{i}, Z_{i j}, W_{i}$. Assume that a minimal free resolution of $k$ is given as in (2.8). Since $I \cdot \operatorname{Tor}_{1}^{T}(k, R)=0$, it can be easily seen that $\sum_{i=1}^{d} c_{i} x_{i}=0\left(c_{i} \in R, 1 \leqq i \leqq d\right)$ implies $I\left(c_{1}, c_{2}, \cdots, c_{d}\right) \in R^{d^{\prime}} \cdot A$. Let $a_{1}, a_{1}, \cdots, a_{l}$ be in $I$. We want to prove $a_{1} a_{2} \cdots a_{l}=0$. For this, looking at the $(l+1)$-th column of the equation

$$
\boldsymbol{E}=\sum_{i=1}^{l} W_{i}\left(\boldsymbol{Y}_{i}, \boldsymbol{x}\right) \text {, }
$$

we see that $W_{l} x=0$ (i.e. $\sum_{i=1}^{d} w_{i}^{(l)} x_{i}=0$ ). Then it follows from the above remark that $a_{1} W_{l} \in R^{d}$ is an $R$-linear combination of rows of $A$. Combining this with the equation $A\left(\boldsymbol{Y}_{l}, \boldsymbol{x}\right)=\sum_{j=1}^{l-1} \boldsymbol{Z}_{i-1 j}\left(\boldsymbol{Y}_{j}, \boldsymbol{x}\right)$, one obtains that $a_{1} W_{l}\left(Y_{l}, \boldsymbol{x}\right)=\sum_{j=1}^{l-1} \boldsymbol{V}_{j}\left(\boldsymbol{Y}_{j}, \boldsymbol{x}\right)$ for some $\boldsymbol{V}_{j} \in R^{d}(1 \leqq j \leqq l-1)$. Hence by $\left(^{*}\right)$ we get

$$
a_{1} E=\sum_{j=1}^{l-1} W_{j}^{\prime}\left(\boldsymbol{Y}_{j}, \boldsymbol{x}\right)
$$

where $W_{j}^{\prime}=a_{1} W_{j}+V_{j}(1 \leqq j \leqq l-1)$. Looking at the $l$-th column of $(* *)$, we have $W_{l-1}^{\prime} x=0$. The same argument as above shows that

$$
a_{1} a_{2} E=\sum_{j=1}^{l-2} W_{j}^{\prime \prime}\left(Y_{j}, \boldsymbol{x}\right)
$$

for some $W_{j}^{\prime \prime} \in R^{d}(1 \leqq j \leqq l-2)$. Iterating this, we arrive in the end at $a_{1} a_{2} \cdots a_{l} \boldsymbol{E}=0$. Hence $a_{1} a_{2} \cdots a_{l}=0$ in $R$.

\section{§3. Big Cohen-Macaulay modules}

Let $T$ be a (Noetherian, not necessarily Artinian in this section) local 
ring with maximal ideal $m$ and let $R$ be a $T$-algebra. We make the following definition.

(3.1) Definition. $R$ is said to be a very rich T-algebra if, for any m-primary ideal $\mathfrak{q}$ of $T, R / \mathfrak{q} R$ is a rich $T / \mathfrak{q}$-algebra.

(3.2) Remark. (i) If $T$ is Artinian, then $R$ is a very rich $T$-algebra if and only if it is rich. This can be easily verified from (2.5) (a-1).

(ii) Let $\left\{\mathfrak{q}_{i} \mid i \in \mathbb{N}\right\}$ be a set of m-primary ideals of $T$ such that $\mathfrak{q}_{1} \supset \mathfrak{q}_{2}$ $\supset \mathfrak{q}_{3} \supset \ldots$ and $\bigcap_{i=1}^{\infty} \mathfrak{q}_{i} \hat{T}=0$. Then a $T$-algebra $R$ is a very rich $T$-algebra if and only if $R / \mathfrak{q}_{i} R$ is a rich $T / \mathfrak{q}_{i}$-algebra for each $i \in \mathbb{N}$.

Proof. By definition "only if" part is trivial. To prove "if" part, let $\mathfrak{q}$ be an arbitrary m-primary ideal of $T$. By $[\mathrm{N}$; Theorem (30.1)] one can find an integer $i$ such that $\mathfrak{q}$ contains $\mathfrak{q}_{i}$. Then $R / \mathfrak{q} R \cong R / \mathfrak{q}_{i} \otimes_{T / \mathfrak{q} i} T / \mathfrak{q}$ is a rich $T / q$-algebra by $(2.5)(a-1)$.

The following is the direct consequence of (2.5).

(3.3) Lemma. (a) Consider the diagram of rings;

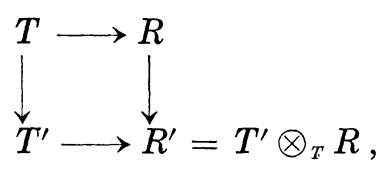

where $T$ and $T^{\prime}$ are local rings with maximal ideals $\mathrm{m}$ and $\mathrm{m}^{\prime}$ respectively, such that $\mathrm{m} T^{\prime}$ is a (proper) $\mathrm{m}^{\prime}$-primary ideal.

(a-1) If $R$ is a very rich T-algebra, then $R^{\prime}$ is a very rich $T^{\prime}$-algebra.

(a-2) If $T^{\prime}$ is flat over $T$, then the converse of (a-1) is also true.

(b) Consider ring homomorphisms $T \rightarrow R \rightarrow S$.

(b-1) If $S$ is a very rich T-algebra, then $R$ is also a very rich T-algebra.

(b-2) Assume that $R$ is also a local ring with maximal ideal $\mathfrak{n}$ such that $\mathrm{m} R$ is a (proper) n-primary ideal. If $S$ is a very rich $R$-algebra and if $R$ is a very rich $T$-algebra, then $S$ is a very rich $T$-algebra.

We can also generalize Proposition (2.6) into the following form.

(3.4) Proposition. (i) If a ring homomorphism $T \rightarrow R$ has a ring retraction, then $R$ is a very rich T-algebra.

(ii) If $R$ is a flat T-algebra, then $R$ is a very rich T-algebra.

(iii) If $R$ is a very rich T-algebra which is finitely generated as a $T$ module, then $T$ is a direct summand of $R$ as a T-module. In particular, the natural homomorphism $T \rightarrow R$ is pure. 
Proof. (i) and (ii) are clear from (2.6) (i) (ii) respectively. To prove (iii) we first remark that we may assume that $T$ is a complete local ring. For this, note that $T$ is a direct summand of $R$ if and only if the completion $\hat{T}$ of $T$ is a direct summand of $\hat{T} \otimes_{T} R$ by $\left[\mathrm{H}_{2} ;\right.$ Lemma 1]. On the other hand, it is easily observed by the assumption that $\hat{T} \otimes_{T} R$ is a very rich $\hat{T}$-algebra. Thus one can assume that $T$ is complete. Then NorthcottRees theorem ([NR; Theorem 6] or $\left[\mathrm{Y}_{2}\right.$; Theorem]) assures that there is a suquence $\left\{\mathfrak{q}_{i} \mid i \in \mathbb{N}\right\}$ of irreducible $\mathfrak{m}$-primary ideals which is cofinal with the power of $\mathfrak{m}$. Since $T / \mathfrak{q}_{i}$ is a direct summand of $R / \mathfrak{q}_{i} R$ for $i \in \mathbb{N}$ by (2.6) (iii), $\left[\mathrm{H}_{2}\right.$; Remark 2] shows that $T$ is a direct summand of $R$.

Before proceeding to the next, we recall some notation: Let $R$ be a local ring with maximal ideal $\mathfrak{n}$ and let $M$ be a (not necessarily finitely generated) $R$-module. For a system of parameters $\boldsymbol{x}=\left\{x_{1}, x_{2}, \cdots, x_{d}\right\}$ for $R, M$ is called a big Cohen-Macaulay module with respect to $x$ if

$$
\left(x_{1}, x_{2}, \cdots, x_{i}\right) M: x_{i+1} R=\left(x_{1}, x_{2}, \cdots, x_{i}\right) M(0 \leqq i \leqq d-1)
$$

and $\mathfrak{n} M \neq M . \quad M$ is a balanced big Cohen-Macaulay module over $R$ if it is a big Cohen-Macaulay module with respect to any system of parameters for $R$.

Considerable progress on the existence of big Cohen-Macaulay modules was made by $\mathrm{M}$. Hochster in $\left[\mathrm{H}_{1}\right]$. He actually proved that there exists a big Cohen-Macaulay module with respect to a given system of parameters for an equicharacteristic local ring. However the existence problem of such modules is still open in general. On the other hand, J. R. Strooker and J. Bartijn was able to prove that if $R$ has a big Cohen-Macaulay module with respect to some system of parameters, then $R$ has a balanced one. More precisely the following is proved.

(3.5) [S; (13.1.8)]. If $M$ is a big Cohen-Macaulay module with respect to some system of parameters for $R$, then its $n$-adic completion $\hat{M}$ is always a balanced big Cohen-Macaulay module over $R$. In particular, any big Cohen-Macaulay module which is complete in $n$-adic topology is balanced.

One of our main results in this paper is the following

(3.6) Theorem. Let $(T, \mathfrak{m}) \rightarrow(R, \mathfrak{n})$ be a local homomorphism of local rings where $T$ is a regular local ring and $R$ is a finite $T$-module such that $\operatorname{dim}(T)=\operatorname{dim}(R)=d$. Then the following conditions are equivalent.

(i) $R$ is a very rich $T$-algebra. 
(ii) There exists a balanced big Cohen-Macaulay module over $R$.

Proof. We first prove (ii) $\Rightarrow$ (i). Let $M$ be an $R$-module which is a balanced big Cohen-Macaulay module, and let $x=\left\{x_{1}, x_{2}, \cdots, x_{d}\right\}$ be a regular system of parameters of $T$. Set

$$
\begin{aligned}
T_{n} & =T /\left(x_{1}^{n}, x_{2}^{n}, \cdots, x_{d}^{n}\right) T, \quad R_{n}=R /\left(x_{1}^{n}, x_{2}^{n}, \cdots, x_{d}^{n}\right) R, \\
M_{n} & =M /\left(x_{1}^{n}, x_{2}^{n}, \cdots, x_{d}^{n}\right) M
\end{aligned}
$$

and $\mathfrak{m}_{n}=\mathfrak{m} T_{n}$ for any integer $n$. It is sufficient from (3.2) (ii) to prove that $M_{n}$ is an $T_{n}$-injective $R_{n}$-module. For this we prove that every $T_{n}$ homomorphism $\mathfrak{m}_{n} \rightarrow M_{n}$ can be extended to a $T_{n}$-homomorphism $T_{n} \rightarrow M_{n}$. (This will imply that $\operatorname{Ext}_{T_{n}}^{1}\left(k, M_{n}\right)=0$ where $k=T / \mathfrak{m}$, hence $M_{n}$ is $T_{n}$ injective.) Note that, for $n \geqq 3$, the minimal free resolution of $k$ as a $T_{n}$-module is given by the following;

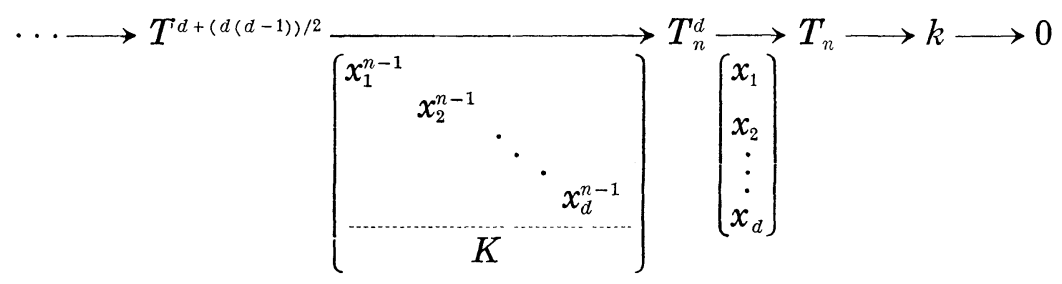

where $K$ is the Koszul relation of $\left\{x_{1}, x_{2}, \cdots, x_{d}\right\}$, i.e. $K$ consists of $d(d-1) / 2$ rows which are in the form

$$
\left(0, \cdots, 0, x_{i}, 0, \cdots,-x_{j}, 0, \cdots, 0\right)(1 \leqq j<i \leqq d) .
$$

Hence by Lemma (2.18) one sees that giving a $T_{n}$-homomorphism of $\mathfrak{m}_{n}$ to $M_{n}$ is equivalent to giving an element $\left(y_{1}, y_{2}, \cdots, y_{d}\right)$ of $M_{n}^{d}$ which satisfies $x_{i} y_{j}=x_{j} y_{i}(i \neq j)$ and $x_{i}^{n-1} y_{i}=0(1 \leqq i \leqq d)$. Thus it suffices to show the following.

(3.7) If $\left(y_{1}, y_{2}, \cdots, y_{d}\right) \in M_{n}^{d}$ satisfies $x_{i} y_{j}=x_{j} y_{i}(i \neq j)$ and $x_{i}^{n-1} y_{i}=0$ $(1 \leqq i \leqq d)$, then there exists $y \in M_{n}$ such that $y_{i}=x_{i} y(1 \leqq i \leqq d)$.

For any $j(j=1,2, \cdots, d)$ we prove a more general assertion.

(3.7.j) Assume that $\left(y_{1}, y_{2}, \cdots, y_{d}\right) \in M_{n}^{d}$ satisfies the same condition as in (3.7). Then for any sequence of $j$ integers $\left\{i_{1}, i_{2}, \cdots, i_{j}\right\}\left(1 \leqq i_{1}<i_{2}<\right.$ $\left.\cdots<i_{j} \leqq d\right)$, there exists an element $z\left(i_{1}, i_{2}, \cdots, i_{j}\right)$ of $M_{n}$ such that $y_{k}=$ $x_{k} \cdot z\left(i_{1}, i_{2}, \cdots, i_{j}\right)$ for $k \in\left\{i_{1}, i_{2}, \cdots, i_{j}\right\}$.

If (3.7.d) is true, then $y=z(1,2, \cdots, d)$ will satisfy (3.7).

We prove (3.7.j) by the induction on $j$. Since a sequence of any power 
of $x_{1}, x_{2}, \cdots, x_{d}$ forms a regular sequence on $M$ in any order, and since $x_{i}^{n-1} y_{i}=0$ in $M_{n}$, it follows that $y_{i} \in x_{i} M_{n}$ for $i=1,2, \cdots, d$. Thus there is a $z_{i} \in M_{n}$ such that $y_{i}=x_{i} z_{i}$ for each $i=1,2, \cdots, d$. This proves (3.7.1).

Assume that (3.7. $j-1)$ is true. Take $\left\{i_{1}, i_{2}, \cdots, i_{j}\right\}$ such that $1 \leqq i_{1}<i_{2}$ $<\cdots<i_{j} \leqq d$. By the induction hypothesis there are $z\left(i_{1}, i_{2}, \cdots, i_{j-1}\right)$ and $z\left(i_{1}, \cdots, i_{j-2}, i_{j}\right)$ in $M_{n}$ satisfying

$$
x_{i_{j-1}} x_{i_{j}}\left(z\left(i_{1}, i_{2} \cdots, i_{i-1}\right)-z\left(i_{1}, \cdots, i_{j-2}, i_{j}\right)\right)=x_{i_{j}} y_{i_{j-1}}-x_{i_{j-1}} y_{i_{j}}=0
$$

and

$$
x_{i_{k}}\left(z\left(i_{1}, i_{2}, \cdots, i_{j-1}\right)-z\left(i, \cdots, i_{j-2}, i_{j}\right)=0\right.
$$

if $1 \leqq k \leqq j-2$.

Combining this with the fact that $M$ is a balanced big Cohen-Macaulay module over $R$, we get

$$
\begin{aligned}
& z\left(i_{1}, i_{2}, \cdots, i_{j-1}\right)-z\left(i_{1}, \cdots, i_{j-2}, i_{j}\right) \in\left[0: x_{i_{j-1}} x_{i_{j}}\right]_{M_{n}} \cap \bigcap_{k=1}^{j-2}\left[0: x_{i_{k}}\right]_{M_{n}} \\
& \quad=\left(x_{i_{j-1}}^{n-1}, x_{i_{j}}^{n-1}\right) M_{n} \cap \bigcap_{k=1}^{j-2} x_{i_{k}}^{n-1} M_{n} \\
& \quad=\left(x_{i_{1}}^{n-1} x_{i_{2}}^{n-1} \cdots x_{i_{j-1}}^{n-1}, x_{i_{1}}^{n-1} x_{i_{2}}^{n-1} \cdots x_{i_{j-2}}^{n-1} x_{i_{j}}^{n-1}\right) M_{n} .
\end{aligned}
$$

Hence there are $z^{\prime}$ and $z^{\prime \prime}$ in $M_{n}$ such that

$$
\begin{aligned}
& z\left(i_{1}, i_{2}, \cdots, i_{j-1}\right)+\left(x_{i_{1}}^{n-1} x_{i_{2}}^{n-1} \cdots x_{i_{j-1}}^{n-1}\right) z^{\prime} \\
& \quad=z\left(i_{1}, \cdots, i_{j-2}, i_{j}\right)+\left(x_{i_{1}}^{n-1} \cdots x_{i_{j-2}}^{n-1} x_{i_{j}}^{n-1}\right) z^{\prime \prime} .
\end{aligned}
$$

Denoting the both side of this equality by $z\left(i_{1}, i_{2}, \cdots, i_{j}\right)$, we see that $x_{i_{k}} z\left(i_{1}, i_{2}, \cdots, i_{j}\right)=y_{i_{k}}$ for any $k=1,2, \cdots, j$. This proves (3.7.j), hence proves (ii) $\Rightarrow$ (i) in Theorem (3.6).

We next prove (i) $\Rightarrow$ (ii) in Theorem (3.6). The following argument is of particular importance and contains the central idea of this paper.

Let $x_{1}, x_{2}, \cdots, x_{d}$ be a regular system of parameters of $T$ and $T_{n}=$ $T /\left(x_{1}^{n}, x_{2}^{n}, \cdots, x_{d}^{n}\right) T$ and $R_{n}=R /\left(x_{1}^{n}, x_{2}^{n}, \cdots, x_{d}^{n}\right) R$ for any integer $n$ as above. By the assumption there exists a $T_{n}$-injective $R_{n}$-module $M_{n}$ for each $n$. Notice that $M_{n}$ is free over $T_{n}$ since $T_{n}$ is a Gorenstein ring of dimension 0. Take an arbitrary non-principal ultrafilter on $\mathbb{N}$ and denote by $\tilde{M}$ the separated ultraproduct of the $R_{n}$-module $M_{n}$. Note that $\tilde{M}$ is a module over $\tilde{R}^{\mathrm{N}}=\tilde{\prod}_{n \in \mathbb{N}} R_{n}$ by $(1.15)$, hence a module over $R$. We prove the following

(3.8) Claim. $\tilde{M}$ is a balanced big Cohen-Macaulay module over $R$. 
We know from (1.8) (iv) and (1.11) that $\tilde{M}$ is a non-trivial, complete and separated module. It is hence sufficient from (3.5) that $\left\{x_{1}, x_{2}, \cdots, x_{d}\right\}$ forms a regular sequence on $\tilde{M}$. For this purpose assume the equality;

$$
x_{1}\left(s_{i}\right)_{i \in \mathbb{N}}+\cdots+x_{k}\left(t_{i}\right)_{i \in \mathbb{N}}+x_{k+1}\left(u_{i}\right)_{\tilde{i} \in \mathbb{N}}=0
$$

where $0 \leqq k<d$ and $\left(s_{i}\right)_{i \in \mathbb{N}}, \cdots,\left(t_{i}\right)_{i \in \mathbb{N}}$ and $\left(u_{i}\right)_{i \in \mathbb{N}}$ are in $\tilde{M}$. We want to show that $\left(u_{i}\right)_{i \in \mathbb{N}} \in\left(x_{1}, \cdots, x_{k}\right) \tilde{M}$. By definition for any integer $n$ it holds that

$$
x_{1} s_{i}+\cdots+x_{k} t_{i}+x_{k+1} u_{i} \in\left(x_{1}^{n}, x_{2}^{n}, \cdots, x_{d}^{n}\right) M_{\imath}
$$

for a.a. $i \in \mathbb{N}$. Hence there are $s_{i}^{\prime}, \cdots, t_{i}^{\prime}, u_{i}^{\prime}, v_{i}^{\prime}, \cdots, w_{i}^{\prime} \in M_{i}$ for those $i \in \mathbb{N}$ such that

$$
\begin{aligned}
& x_{1}\left(s_{i}+x_{1}^{n-1} s_{\imath}^{\prime}\right)+\cdots+x_{k}\left(t_{\imath}+x_{k}^{n-1} t_{i}^{\prime}\right)+x_{k+1}\left(u_{i}+x_{k+1}^{n-1} u_{i}^{\prime}\right) \\
& +x_{k+2}^{n} v_{i}^{\prime}+\cdots+x_{d}^{n} w_{i}^{\prime}=0 .
\end{aligned}
$$

Since $M_{i}$ is $T_{i}$-free, one sees that

$$
\begin{aligned}
u_{i}+x_{k+1}^{n-1} u_{i}^{\prime} & \in\left[\left(x_{1}, \cdots, x_{k}, x_{k+2}^{n}, \cdots, x_{d}^{n}\right) M_{i}: x_{k+1}\right]_{M_{i}} \\
& =\left(x_{1}, \cdots, x_{k}, x_{k+1}^{2-1}, x_{k+2}^{n}, \cdots, x_{d}^{n}\right) M_{i}
\end{aligned}
$$

for those $i \in \mathbb{N}$. This implies that

$$
u_{i} \in\left(x_{1}, \cdots, x_{k}, x_{k+1}^{n-1}, x_{k+2}^{n}, \cdots, x_{d}^{n}\right) M_{i}
$$

for a.a. $i \in \mathbb{N}$.

Denote $\bar{M}_{i}=M_{i} /\left(x_{1}, \cdots, x_{k}\right) M_{i}$ and denote by $\bar{u}_{i}$ the natural image of $u_{i}$ in $\bar{M}_{i}$. Then the above argument shows that, for any integer $n, \bar{u}_{i} \in$ $\left(x_{k+1}^{n-1}, x_{k+2}^{n}, \cdots, x_{d}^{n}\right) \bar{M}_{i}$ for a.a. $i \in \mathbb{N}$. This implies that $\left(\bar{u}_{i}\right)_{i \in \mathbb{N}}=0$ in $\tilde{\prod}_{i \in \mathbb{N}} \bar{M}$. Consider the trivial exact sequence; $0 \rightarrow\left(x_{1}, \cdots, x_{k}\right) M_{i} \rightarrow M_{i} \rightarrow \bar{M}_{i} \rightarrow 0$ for all $i \in \mathbb{N}$. Here we remark that

$$
\begin{aligned}
& \mathfrak{m}^{n} M_{i} \cap\left(x_{1}, \cdots, x_{k}\right) M_{i}=\mathfrak{m}^{n-1}\left(x_{1}, \cdots, x_{k}\right) M_{i} \\
& \text { for all } n \text { and } i \in \mathbb{N} \text {. In particular, } a_{T_{i}}\left(\left(x_{1}, \cdots, x_{k}\right) M_{i}, M_{i}\right)=1 \\
& \text { for all } i \in \mathbb{N} \text {. }
\end{aligned}
$$

If this is correct, then by Theorem (1.18) and (1.8) (v) we see that

$$
0 \longrightarrow \prod_{i \in \mathbb{N}}\left(x_{1}, \cdots, x_{k}\right) M_{i} \longrightarrow \tilde{\prod}_{i \in \mathbb{N}} M_{i} \longrightarrow \tilde{\prod}_{i \in \mathbb{N}} \bar{M}_{i} \longrightarrow 0
$$

is an exact sequence of $\tilde{R}$-modules. Thus $\left(u_{i}\right)_{i \in \mathbb{N}}$ is the image of an element $\left(x_{1} a_{i}+\cdots+x_{k} b_{i}\right)_{i \in \mathbb{N}}$ of $\tilde{\Pi}_{i \in \mathbb{N}}\left(x_{1}, \cdots, x_{k}\right) M_{i}$ and this shows that $\left(u_{i}\right)_{i \in \mathbb{N}}=$ $x_{1}\left(a_{1}\right)_{i \in \mathbb{N}}+\cdots+x_{k}\left(b_{i}\right)_{i \in \mathbb{N}} \in\left(x_{1}, \cdots, x_{k}\right) \tilde{M}$ as desired. 
It remains to prove (3.9). Since $M_{i}$ is a $T_{i}$-free module as remarked above, it is enough to show that, for any integer $n$,

$$
\begin{aligned}
& \mathfrak{m}^{n} T_{i} \cap\left(x_{1}, \cdots, x_{k}\right) T_{i}=\mathfrak{m}^{n-1}\left(x_{1}, \cdots, x_{k}\right) T_{i} \\
& \text { for all } i \in \mathbb{N} \text { and } 1 \leqq k<d .
\end{aligned}
$$

We prove this by the induction on $n$. Since it is trivially correct when $n=1$, we assume that $n \geqq 2$ and (3.9. $n-1)$ is true. Then one observes by $(3.9 . n-1)$ that

$$
\mathfrak{m}^{n} T_{i} \cap\left(x_{1}, \cdots, x_{k}\right) T_{i} \subset \mathfrak{m}^{n-2}\left(x_{1}, \cdots, x_{k}\right) T_{i} \cap \mathfrak{m}^{n} T_{i} .
$$

Thus to prove (3.9.n) it is sufficient to show that

$$
\begin{aligned}
\mathfrak{m}^{n-2}\left(x_{1}, \cdots, x_{k}\right) T_{i} & \cap \mathfrak{m}^{n} T_{i} \subset \mathfrak{m}^{n-1}\left(x, \cdots, x_{k}\right) T_{i} \\
& \text { for all } i \in \mathbb{N} \text { and } k(1 \leqq k<d) .
\end{aligned}
$$

We prove (3.10) by induction on $k$. For this, we denote $G_{i}=\operatorname{gr}_{\mathrm{m}_{i}}\left(T_{i}\right)$ the associated graded ring of $T_{i}$ with respect to $m_{i}$. For the regularity of $T$, it can be seen that $G_{i}=(T / \mathfrak{m})\left[X_{1}, X_{2}, \cdots, X_{d}\right] /\left(X_{1}^{i}, X_{2}^{i}, \cdots, X_{d}^{i}\right)$ where the image of $X_{i}$ in $G_{i}$ respresents the initial form of $x_{i}$. Now assume that $a x_{1} \in \mathfrak{m}^{n} T_{i}$ and $a \in \mathfrak{m}^{n-2} T_{i}-\mathfrak{m}^{n-1} T_{i}$. If we denote by $c(i)$ the initial form of $a$ in $G_{i}$, then $c(i) \in X_{1}^{i-1} G_{i}$ because of $c(i) X_{1}=0$. This means that $a \in x_{1}^{i-1} T_{i}$ $+\mathfrak{m}^{n-1} T_{i}$ hence $\operatorname{ax}_{1} \in \mathfrak{m}^{n-1} x_{1} T_{i}$. This proves (3.10) in the case $k=1$.

Assume that $y=\sum_{j=1}^{k} a_{j} x_{j} \in \mathfrak{m}^{n} T_{i}$ where $a_{i} \in \mathfrak{m}^{n-2} T_{i}(1 \leqq i \leqq k)$. If $a_{k} \in \mathfrak{m}^{n-1} T_{i}$, then $y-a_{k} x_{k} \in \mathfrak{m}^{n-2}\left(x_{1}, \cdots, x_{k-1}\right) T_{i} \cap \mathfrak{m}^{n} T_{i}=\mathfrak{m}^{n-1}\left(x_{1}, \cdots, x_{k-1}\right) T_{i}$ by the induction hypothesis. Hence $y \in \mathfrak{m}^{n-1}\left(x_{1}, \cdots, x_{k}\right) T_{i}$. Thus we may assume that $a_{k} \notin \mathfrak{m}^{n-1} T_{i}$. Let $c_{j}(i)$ be the initial form of $a_{j}$ in $G_{i}$. Note that $c_{k}(i)$ has degree $n-2$. Since $\sum_{j=1}^{k} c_{j}(i) X_{j}=0$, we see that $c_{k}(i) \in$ $\left(X_{1}, \cdots, X_{k-1}, X_{k}^{i-1}\right) G_{i}$, that is,

$$
a_{k}=b_{1} x_{1}+\cdots+b_{k-1} x_{k-1}+b_{k} x_{k}^{i-1}+c
$$

for some $b_{j} \in \mathfrak{m}^{n-3} T_{i}(1 \leqq j<k), b_{k} \in \mathfrak{m}^{n-i+1} T_{i}$ and $c \in \mathfrak{m}^{n-1} T_{i}$. Hence $y=$ $\sum_{j=1}^{k-1}\left(a_{j}+b_{j} x_{k}\right) x_{j}+c x_{k}$, and it follows by the induction hypothesis that

$$
y-c x_{k} \in \mathfrak{m}^{n-2}\left(x_{1}, \cdots, x_{k-1}\right) T_{i} \cap \mathfrak{m}^{n} T_{i} \subset \mathfrak{m}^{n-1}\left(x_{1}, \cdots, x_{k-1}\right) T_{i},
$$

thus we get $y \in \mathfrak{m}^{n-1}\left(x_{1}, \cdots, x_{k}\right) T_{i}$. This establishes (3.10), hence completes the proof of Theorem (3.6).

Though we have proved Theorem (3.6), we must accept the fact that the existence problem of big Cohen-Macaulay modules is still in the dark. 
However once we know the existence, we are able to construct modules which enjoy stronger conditions. Precisely we can prove

(3.11) THEOREM. Let $T$ be a complete regular local ring containing a field and let $R$ be the integral closure of $T$ in the algebraic closure $\bar{Q}$ of the quotient field $Q$ of $T$. Then there exists an $R$-module $M$ such that, for any finite $T$-algebra $S$ which lies between $T$ and $R$ and for any system of parameters $\boldsymbol{x}$ for $S, M$ is a big Cohen-Macaulay module over $S$ with respect to $\boldsymbol{x}$.

Before proceeding to the proof we should notice some remarks.

(3.12) Let $N$ be the set of field extensions $K$ such that $Q \subset K \subset \bar{Q}$ and $[K: Q]<\infty$ and let $S_{K}$ be the integral closure of $T$ in $K$ for $K \in N$. Since $T$ is a complete local ring, $S_{K}$ is a local ring which is finite over $T$ for any $K \in N$. (See [N].) The set $\left\{S_{K} \mid K \in N\right\}$ forms an inductive system ordered by inclusion. One can easily verify $R=\varliminf_{K \in N} S_{K}$, hence in particular $R$ is a quasi-local ring. Since $T$ is a Noetherian ring, $\bar{Q}$ is an infinite extension of $Q$. Thus one can find a countable set of elements $\left\{x_{i} \mid i \in \mathbb{N}\right\}$ in $\bar{Q}$ satisfying $Q\left(x_{1}\right) \subseteq Q\left(x_{1}, x_{2}\right) \subseteq \cdots \sqsubseteq Q\left(x_{1}, x_{2}, \cdots, x_{i}\right) \subseteq Q\left(x_{1}, x_{2}\right.$, $\left.\cdots, x_{i+1}\right) \cdots$ If we denote $A(S)=\{K \in N \mid K \supset S\}$ for a subset $S$ of $\bar{Q}$, then $\{A(S) \mid S$ is a finite subset of $\bar{Q}\}$ forms a filter on $N$, hence there is an ultrafilter $\widetilde{F}$ on $N$ containing those $A(S)$ for finite $S$. Since $A\left(x_{1}, x_{2}, \cdots, x_{i}\right)$ $\in \mathfrak{F}$ for any $i \in \mathbb{N}$ and since

$$
\bigcap_{i=1}^{\infty} A\left(x_{1}, x_{2}, \cdots, x_{i}\right)=\phi,
$$

it follows that $\widetilde{\Im}$ is an $\omega$-incomplete ultrafilter on $N$. (See (1.3).) Thus one can consider the separated ultraproduct $\tilde{\Pi}_{K \in N} S_{K}$ as T-modules (not as $S_{K^{-}}$ modules). Denote it by $\tilde{S}$. Then $\tilde{S}$ is naturally a $T$-algebra. For any element $x$ of $R, x$ belongs to $S_{K}$ for a.a. $K \in N$, hence $x$ gives the element $(x)_{i \in N}$ of $\tilde{S}$ by (1.8) (iii). This defines a map $f: R \rightarrow \tilde{S}$. It is easily seen that $f$ is a $T$-algebra homomorphism. In order to prove Theorem (3.11) it is hence sufficient to show that there is an $\tilde{S}$-module $\tilde{M}$ such that, for any $K \in N, \tilde{M}$ is a big Cohen-Macaulay module over $S_{K}$.

Proof of Theorem (3.11). We shall construct such an $\tilde{S}$-module $\tilde{M}$. Since each $S_{K}$ is a local ring containing a field, Hochster's theorem $\left[\mathrm{H}_{1}\right.$; Theorem (5.1)] and (3.5) show that there is a balanced big Cohen-Macaulay module over $S_{K}$. We denote it by $M_{K}$ for $K \in N$, and denote by $\tilde{M}$ the 
separated ultraproduct of the $M_{K}$ as $T$-modules (not as $S_{K}$-modules). Note that $\tilde{M}$ is a module over $\tilde{S}$, hence a module over $S_{K}$ for any $K \in N$. Since $\tilde{M}$ is a separated and complete $T$-module by (1.11) and since $S_{K}$ is finite over $T, \tilde{M}$ is also separated and complete as an $S_{K}$-module. We shall prove that this $\tilde{M}$ satisfies the required condition. For this, it suffices by (3.5) to show that $\boldsymbol{x}=\left\{x_{1}, x_{2}, \cdots, x_{d}\right\}$ is a regular sequence on $\tilde{M}$, where $\boldsymbol{x}$ is a regular system of parameters of $T$. This is done in the following course.

Let $\sum_{i=1}^{k+1} x_{i}\left(y_{K}(i)\right)_{\tilde{K} \in N}=0$ where $1 \leqq k<d$ and $\left(y_{K}(i)\right)_{\tilde{K} \in N} \in \tilde{M}(i=1,2$, $\cdots, k)$. We would like to show that $\left(y_{K}(k+1)\right)_{\tilde{K} \in N} \in\left(x_{1}, x_{2}, \cdots, x_{k}\right) \tilde{M}$. By definition it holds that, for any integer $n, \sum_{i=1}^{k+1} x_{i} y_{K}(i) \in\left(x_{1}^{n}, x_{2}^{n}, \cdots, x_{d}^{n}\right) M_{K}$ for a.a. $K \in N$. Since $\boldsymbol{x}$ forms a regular sequence on $M_{K^{\prime}}$ for any $K \in N$, we easily see that

$$
y_{K}(k+1) \in\left(x_{1}, \cdots, x_{k}, x_{k+1}^{n-1}, x_{k+2}^{n}, \cdots, x_{d}^{n}\right) M_{K}
$$

for those $K \in N$.

If we denote $\bar{M}_{K}=M_{K} /\left(x_{1}, \cdots, x_{k}\right) M_{K}$ and denote by $\bar{y}_{K}(i)$ the natural image of $y_{K}(i)$ in $\bar{M}_{K}$, then the above shows that $\left(\bar{y}_{K}(k+1)\right)_{\tilde{K} \in N}=0$ in $\tilde{\Pi}_{K \in N} \bar{M}_{K}$.

Consider the trivial exact sequence;

$$
0 \longrightarrow\left(x_{1}, \cdots, x_{k}\right) M_{K} \longrightarrow M_{K} \longrightarrow \bar{M}_{K} \longrightarrow 0
$$

for all $K \in N$. Here one can verify the following;

$$
\mathfrak{m}^{n} M_{K} \cap\left(x_{1}, \cdots, x_{k}\right) M_{K}=\mathfrak{m}^{n-1}\left(x_{1}, \cdots, x_{k}\right) M_{K}
$$

for all $n \in \mathbb{N}$ and $K \in N$. (Since $\left\{x_{1}, \cdots, x_{k}\right\}$ is a regular sequence on $M_{K}$, [EGA; (15.1.9)] shows that $\operatorname{gr}_{\mathrm{m}}\left(M_{K}\right) \cong\left(M_{K} / \mathrm{m} M_{K}\right)\left[X_{1}, \cdots, X_{k}\right]$. Then the claim can be proved in the same manner as in (3.9). So we leave the detail to the reader.) In particular, $a_{T_{i}}\left(\left(x_{1}, \cdots, x_{k}\right) M_{K}, M_{K}\right)=1$ for all $K \in N$. Therefore Theorem (1.18) gives the exact sequence;

$$
0 \longrightarrow \tilde{\prod}_{K \in N}\left(x_{1}, \cdots, x_{k}\right) M_{K} \longrightarrow \tilde{M} \longrightarrow \tilde{\prod}_{K \in N} \bar{M}_{K} \longrightarrow 0
$$

Hence $\left(y_{K}(k+1)\right)_{\tilde{K} \in N}$ is the image of an element of $\tilde{\Pi}_{K \in N}\left(x_{1}, \cdots, x_{k}\right) M_{K}$, thus it is in $\left(x_{1}, \cdots, x_{k}\right) \tilde{M}$. This completes the proof of Theorem (3.11).

\section{REFERENCES}

[B] H. Bass, On the ubiquity of Gorenstein rings, Math. Z., 82 (1963), 8-28.

[BDLD] J. Becker, J. Denef, L. Lipshits and van den Dries, Ultraproducts and approximation in local rings I, Invent. Math., 51 (1979), 189-203. 
[CE] H. Cartan and S. Eilenberg, Homological Algebra, Princeton, New Jersey, Princeton University Press, 1956.

[EGA] A. Grothendieck and J. Dieudonne, Element de Geometrie Algebrique, Chapter $0_{\text {IV, } 1}$, Inst. Hautes Etudes Sci. Publ. Math., no. 20 (1964).

[F] H.-B. Foxby, A homological theory of complexes of modules, Københavens Univ. Math. Inst., Preprint Series no. 196, September, 1981.

$\left[\mathrm{H}_{1}\right] \mathrm{M}$. Hochster, Topics in the homological theory of modules over commutative rings, C. B. M. S. Regional Conference Series in Math. no. 24, Amer. Math. Soc., Providence, R. I., 1975. ,

$\left[\mathrm{H}_{2}\right] \quad$ - Contracted ideals from integral extension of regular local rings, Nagoya Math. J., 51 (1975), 25-43.

$\left[\mathrm{H}_{3}\right] \longrightarrow$ Cohen-Macaulay modules, Proc. Kansas Commutative Algebra Conference, Lecture Note in Math., vol. 311, Springer Verlag, Berlin-Heiderberg-New York, 1973, 120-152.

$\left[\mathrm{H}_{4}\right] \quad-$, Big Cohen-Macaulay modules and algebras and embeddability in rings of Witt vectors, Queen's papers in Pure Appl. Math., 42 (1975), 106-195.

$\left[\mathrm{H}_{5}\right] \longrightarrow$ Canonical elements in local cohomology modules and the direct summand conjecture, preprint.

[I] B. Iversen, Amplitude inequalities for complexes, Ann. Scient. Éc. Norm. Sup., (4) 10 (1977), 547-558.

[M] E. Matlis, Injective modules over Noetherian rings, Pacific J. Math., 8 (1958), 511-528.

[Ma] H. Matsumura, Commutative Algebra, Benjamin, New York, 1970.

[MP] C. Mateescu and D. Popescu, Ultraproducts and big Cohen-Macaulay modules, preprint.

[N] M. Nagata, Local Rings, Interscience Tracts in Pure nad Appl. Math., no. 13, Interscience, New York, 1962.

[NR] D. G. Northcott and D. Rees, Principal systems, Qurt. J. Math. Oxford, (2) 8 (1957), 119-127.

[PS ${ }_{1}$ C. Peskine and L. Szpiro, Dimension projective finie et cohomologie locale, I.H.E.S. Publ. Math., no. 42, Paris, 1973, 323-395.

[PS $\left.{ }_{2}\right] \quad-$, Syzygy et multiplicités, C. R. Acad. Sci. Paris, Serie A-B 278 (1974), 1421-1424.

$\left[R_{1}\right] \quad P$. Roberts, Two applications of dualizing complexes over local rings, Ann. Scient. Ec. Norm. Sup., (4) 9 (1976), 103-106.

$\left[R_{2}\right]$, Cohen-Macaulay complexes and an analytic proof of the new intersection conjecture, J. Algebra, 66 (1980), 202-225.

[S] J. R. Strooker, The monomial and direct summand conjectures, a draft for Chapter 13 of a book on the homological conjectures, Univ. of Utrecht, June, 1981.

[ $\left.\mathrm{Y}_{1}\right] \quad \mathrm{Y}$. Yoshino, On the homological conjectures of local rings, Proc. of the 9th International Symposium, Div. of Math. of the Taniguchi Foundation, Conference on Commutative Algebra, Katata, September, 1981, 51-56.

[ $\left.\mathrm{Y}_{2}\right] \quad-$, On Northcott-Rees theorem on principal systems, Nagoya Math. J., 95 (1984), 41-50.

Department of Mathematics

Nagoya University

Nagoya 464 Japan 